\title{
Critical non-Abelian vortices and holography for little string theory
}

\author{
E. Ievlev $\oplus^{1,2, *}$ and A. Yung $\oplus^{1,3}$ \\ ${ }^{1}$ National Research Center "Kurchatov Institute”, Petersburg Nuclear Physics Institute, \\ Gatchina, St. Petersburg 188300, Russia \\ ${ }^{2}$ Saint Petersburg State Electrotechnical University, ul. Professora Popova, St. Petersburg 197376, Russia \\ ${ }^{3}$ William I. Fine Theoretical Physics Institute, University of Minnesota, \\ Minneapolis, Minnesota 55455, USA
}

(Received 26 October 2021; accepted 2 December 2021; published 28 December 2021)

\begin{abstract}
It has been shown that non-Abelian vortex string supported in four-dimensional (4D) $\mathcal{N}=2$ supersymmetric QCD (SQCD) with the $\mathrm{U}(2)$ gauge group and $N_{f}=4$ quark flavors becomes a critical superstring. This string propagates in the ten-dimensional space formed by a product of the flat 4D space and an internal space given by a Calabi-Yau noncompact threefold, namely, the conifold. The spectrum of closed string states of the associated string theory was obtained using the equivalence between the critical string on the conifold and the noncritical string on the semi-infinite cigar described by the $\operatorname{SL}(2, \mathbb{R}) / U(1)$ Wess-Zumino-Novikov-Witten model. This spectrum was identified with the spectrum of hadrons in 4D $\mathcal{N}=2$ SQCD. In order to describe effective interactions of these 4D hadrons in this paper, we study correlation functions of normalizable vertex operators localized near the tip of the $\operatorname{SL}(2, \mathbb{R}) / \mathrm{U}(1)$ cigar. We also compare our solitonic string approach to the gauge-string duality to the AdS/CFT-type holography for little string theories. The latter relates off mass-shell correlation functions on the field theory side to correlation functions of non-normalizable vertex operators on the cigar. We show that in most channels the holographic approach works in our theory because normalizable and non-normalizable vertex operators with the same conformal dimension are related due to the reflection from the tip of the cigar. However, we find that holography does not work for lightest hadrons with given baryonic charge.
\end{abstract}

DOI: 10.1103/PhysRevD.104.114033

\section{INTRODUCTION}

Non-Abelian vortices were first found in four-dimensional (4D) $\mathcal{N}=2$ supersymmetric QCD (SQCD) with the gauge group $\mathrm{U}(N)$ and $N_{f} \geq N$ flavors of quark hypermultiplets [1-4]. The non-Abelian vortex string is $1 / 2$ Bogomol'nyi-Prasad-Sommerfield (BPS) saturated and, therefore, has $\mathcal{N}=(2,2)$ supersymmetry on its world sheet. In addition to the four translational moduli characteristic of the Abrikosov-Nielsen-Olesen strings [5], the nonAbelian string carries orientational moduli, as well as the size moduli if $N_{f}>N$ [1-4] (see [6-9] for reviews).

Recently, it was discovered that the non-Abelian solitonic vortex string in $4 \mathrm{D} \mathcal{N}=2$ supersymmetric QCD at strong coupling can become a critical superstring [10]. This was shown to happen in the theory with the $\mathrm{U}(2)$ gauge group, four quark flavors, and the Fayet-Iliopoulos (FI)

\footnotetext{
*ievlevea@thd.pnpi.spb.ru
}

Published by the American Physical Society under the terms of the Creative Commons Attribution 4.0 International license. Further distribution of this work must maintain attribution to the author(s) and the published article's title, journal citation, and DOI. Funded by SCOAP.
[11] parameter $\xi$. Due to the extended supersymmetry, the gauge coupling in the 4D SQCD could be renormalized only at one loop. With the judicial choice of the matter sector, the one-loop renormalization cancels. No dynamical scale parameter $\Lambda$ is generated in $4 \mathrm{D} \mathrm{SQCD}{ }^{1}$

The dynamics of the internal (orientational and size) moduli of the non-Abelian vortex string in the case at hand $\left(N=2, N_{f}=4\right)$ is described by the so-called two-dimensional (2D) weighted $\mathbb{C P}$ sigma model, which we denote as $\mathbb{W C P}(2,2)$, see Sec. II B below. Its $\beta$ function vanishes, and the dimension of the target space of the $\mathbb{W C C P}(2,2)$ model is equal to six [10]. Thus, in this case, the target space of the world-sheet theory of the non-Abelian vortex string is ten dimensional (10D)as required for a superstring to become critical. It has a structure $\mathbb{R}^{4} \times Y_{6}$, where $Y_{6}$ is a noncompact six-dimensional Calabi-Yau manifold, the resolved conifold $[12,13]$. This allows one to apply the string theory for the calculation of the spectrum of closed string states [14]. This spectrum was interpreted as a spectrum of hadrons in 4D $\mathcal{N}=2$ SQCD. The vortex string at hand was identified as the superstring theory of type IIA [14].

\footnotetext{
${ }^{1}$ However, conformal invariance of 4D SQCD is broken by the Fayet-Iliopoulos term.
} 
A version of the string-gauge duality for 4D SQCD was proposed [10]; at weak coupling, this theory is in the Higgs phase and can be described in terms of quarks and Higgsed gauge bosons, while at strong coupling hadrons of this theory can be understood as string states formed by the non-Abelian vortex string. Below, we call this approach "solitonic string-gauge duality."

The study of the above vortex string from the standpoint of string theory, with the focus on massless states in four dimensions has been started in $[14,15]$. Generically, most of the massless modes have non-normalizable wave functions over the conifold $Y_{6}$; i.e., they are not localized in 4D and, hence, cannot be interpreted as dynamical states in 4D SQCD. In particular, no massless 4D gravitons or vector fields were found in the physical spectrum in [14]. However, a single massless BPS hypermultiplet in the 4D SQCD was detected at a self-dual point (at strong coupling). It is associated with deformations of a complex structure of the conifold and was interpreted as a composite 4D "baryon". ${ }^{2}$ Later, the existence of this massless hypermultiplet was confirmed using purely field theoretical methods [16].

The low lying massive string states were found in [17], and the corresponding multipet structure with respect to the $4 \mathrm{D} \mathcal{N}=2$ supersymmetry was identified in [18]. To analyze the massive states, a different approach was applied, similar to the one used for little string theories (LSTs), see [19] for a review. This is the equivalence between the critical string on the conifold and noncritical $c=1$ string which contains the Liouville field and a compact scalar at the selfdual radius [20,21]. Generically, the above equivalence is formulated between the critical string on noncompact CalabiYau spaces with isolated singularity on the one hand and noncritical $c=1$ string with the additional GinzburgLandau $\mathcal{N}=2$ superconformal system [21] on the other hand. In the conifold case, this extra Ginzburg-Landau conformal field theory (CFT) is absent [22].

The above $c=1$ Liouville theory has a mirror description [23]. In this formulation, it is given by supersymmetric version of Witten's 2D black hole with a semi-infinite cigar target space [24], which is the $\mathrm{SL}(2, R) / \mathrm{U}(1)$ coset WessZumino-Novikov-Witten (WZNW) theory $[20,21,25,26]$. It can be analyzed by virtue of algebraic methods, and the spectrum of primary operators was computed exactly [25,27-30]. These exact results were used in [17] to obtain a low lying spectrum of hadrons in $\mathcal{N}=24 D$ SQCD. In the string description, 4D SQCD hadrons are associated with normalizable vertex operators localized near the tip of the cigar.

In this paper, we make a next step and apply string theory description to study interactions of 4D hadrons. To this end,

\footnotetext{
${ }^{2}$ If the gauge group is $\mathrm{U}(2)$, as is our case, there are no bona fide baryons. We still use the term baryon because of a particular value of its charge $Q_{B}$ (baryon) $=2$ with respect to the global unbroken $\mathrm{U}(1)_{B}$, see Sec. III C.
}

we calculate correlation functions of normalizable vertex operators in the WZNW $\mathrm{SL}(2, R) / \mathrm{U}(1)$ coset model. These correlation functions were studied by many authors (see, e.g., [31-35] and a review [36]), and we apply already known results to our case.

Another purpose of this paper is to compare the solitonic string-gauge duality to the AdS/CFT approach. The former identify closed string states of the string theory for the solitonic non-Abelian vortex with hadrons of $4 \mathrm{D} \mathcal{N}=2$ SQCD. The latter developed for much broader class of theories relates the open string description of a field theory on the world volume of $N_{b}$ parallel $D$-branes and observables in a theory of a closed string propagating in the background of these branes, see, for example, [37] for a review. We summarize the distinctions of two approaches and suggest that our solitonic string-gauge duality can be thought of as a "no branes" limit of AdS/CFT-type correspondence, $N_{b} \rightarrow 0,{ }^{3}$ for the cigar background. However, holography seems to be a distinctive feature of AdS/CFT correspondence not present in the solitonic string-gauge duality.

To clarify this issue, we compare our solitonic stringgauge duality to the AdS/CFT-type holography for LSTs. In $[21,22,38]$, it was argued that noncritical string theories with the dilaton linear in the Liouville coordinate $\phi$ are holographic. The main example of this behavior is nongravitational LST in the flat six-dimensional space formed by the world volume of $N_{b}$ parallel NS5 branes. The string coupling exponentially goes to zero in the bulk of the space-time (at large $\phi$ ), and nontrivial dynamics (LST) is localized near the branes. Much in the same way as in the AdS/CFT, holography off mass-shell correlation functions on the field theory side (in LST) correspond to string theory correlation functions on the "boundary" at $\phi \rightarrow \infty$. More precisely, off-shell correlation functions in LST correspond to correlation functions of non-normalizable vertex operators on the cigar, see [19] for a review.

In this paper, we test this holography for the string theory of our non-Abelian vortex. We find that in most channels the holographic approach works because normalizable and non-normalizable vertex operators with the same conformal dimension are related due to the reflection from the tip of the cigar.

This relation was already established in [39] in the context of the six-dimensional LST in the background of parallel NS5 branes. Near the pole associated with a physical state with mass $M$, the non-normalizable vertex operator $V^{\text {non-norm }}$ behaves as

$$
V^{\text {non-norm }} \sim \frac{1}{p_{\mu}^{2}+M^{2}} V^{\text {norm }}
$$

\footnotetext{
${ }^{3}$ The validity of our approximation is not related to the large $N_{b}$ limit, see Sec. III A.
} 
where $p_{\mu}$ is the momentum of the field theory state (4D momentum in our case), while $V^{\text {norm }}$ is the normalizable vertex operator of the state with mass $M$. The authors of [39] call these poles Lehmann-Symanzik-Zimmermann (LSZ) poles.

We test the holography relation (1.1) in our theory. First, we confirm that LSZ poles of two-point correlation functions of non-normalizable vertex operators match the mass spectrum found previously $[17,18]$. Next, we consider three-point correlation functions and confirm the holography relation (1.1). The only exceptions are correlation functions of vertex operators which are on the borderline between normalizable and non-normalizable states. These operators correspond to logarithmically normalizable wave functions in our theory and are associated with physical states in 4D SQCD, namely, lightest baryons with given baryon charge. Correlation functions of these operators give scattering amplitudes of the above-mentioned baryons. Although these operators have non-normalizable "partners" with same conformal dimensions, correlation functions of latter operators do not have required LSZ pole structure.

The paper is organized as follows. In Sec. II, we review the non-Abelian string, and its relation to the critical superstring on the conifold. Next, in Sec. III, we review the noncritical $c=1$ string theory and the spectrum of stringy hadrons in 4D SQCD. In Sec. IV, we discuss general features of our solitonic string-gauge duality versus holographic dualities. In Sec. V, we consider two-point correlation functions for operators corresponding to baryons of the underlying $4 \mathrm{D} \mathcal{N}=2$ SQCD. Three-point correlation functions for such operators are studied in Sec. VI. We consider these correlation functions with normalizable as well as non-normalizable operators and test the holography relation. In Sec. VII, we study correlation functions of operators from continuous representations and discuss an interpretation of such states in terms of the 4D $\mathcal{N}=2$ SQCD. Section VIII presents our conclusions. In Appendix A, we cite the three-point function formula in the supersymmetric Liouville theory and apply it for the case of the continuous representations. In Appendix B, we make a comparison of the supersymmetric and nonsupersymmetric Liouville theories, present an idea of factorization, and apply it to the supersymmetric Liouville theory. In Appendix C, we make a consistency check of the two-point correlation function formula. Appendix D contains some useful formulas.

\section{NON-ABELIAN VORTICES}

\section{A. Four-dimensional $\mathcal{N}=2$ SQCD}

As was already mentioned above, non-Abelian vortex strings were first found in $4 \mathrm{D} \mathcal{N}=2 \mathrm{SQCD}$ with the gauge group $\mathrm{U}(N)$ and $N_{f} \geq N$ quark flavors (i.e., hypermultiplets) supplemented by the FI $D$ term $\xi$ [1-4], see, for example, [8] for a detailed review of this theory. Here, we just mention that at weak coupling, $g^{2} \ll 1$, this theory is in the Higgs phase in which the scalar components of the quark multiplets (squarks) develop vacuum expectation values (VEVs). These VEVs breaks the $\mathrm{U}(N)$ gauge group Higgsing all gauge bosons. The Higgsed gauge bosons combine with the screened quarks to form long $\mathcal{N}=2$ multiplets with mass $m \sim g \sqrt{\xi}$.

Since the $\mathcal{N}=2$ SQCD is in the Higgs phase, nonAbelian vortex strings confine monopoles. In the $\mathcal{N}=2$ 4D theory, these strings are 1/2 BPS saturated; hence, their tension is determined exactly by the FI parameter,

$$
T=2 \pi \xi
$$

However, non-Abelian vortices in $\mathrm{U}(N)$ theories are topologically stable and cannot be broken. Therefore, the finite-length strings are closed. In particular, the monopoles cannot be attached to the string end points. In fact, in the $\mathrm{U}(N)$ theories, confined monopoles are junctions of two distinct elementary non-Abelian strings [3,4,40] (see [8] for a review). As a result, in $4 \mathrm{D} \mathcal{N}=2 \mathrm{SQCD}$, we have monopole-antimonopole mesons in which the monopole and antimonopole are connected by two confining strings. In addition, in the $\mathrm{U}(N)$ gauge theory, we can have baryons appearing as a closed "necklace" configurations of $N \times$ (integer) monopoles [8].

Squark VEVs lead to the color-flavor locking phenomenon. The global flavor symmetry group $\mathrm{SU}\left(N_{f}\right)$ and the gauge group $\mathrm{U}(N)$ are broken down, and the resulting global symmetry is

$$
\mathrm{SU}(N)_{C+F} \times \mathrm{SU}\left(N_{f}-N\right) \times \mathrm{U}(1)_{B},
$$

see Ref. [8] for more details.

The unbroken global $\mathrm{U}(1)_{B}$ factor above is identified with a baryonic symmetry. Note that what is usually identified as the baryonic U(1) charge is a part of our $4 \mathrm{D}$ theory gauge group. "Our" $\mathrm{U}(1)_{B}$ is an unbroken by squark VEVs combination of two U(1) symmetries. The first is a subgroup of the flavor $\mathrm{SU}\left(N_{f}\right)$, and the second is the global $\mathrm{U}(1)$ subgroup of $\mathrm{U}(N)$ gauge symmetry.

The stringy monopole-antimonopole mesons as well as monopole baryons with spins $J \sim 1$ are massive. Their masses are determined by the string tension, $\sim \sqrt{\xi}$. They are heavy at weak coupling and decay into perturbative quarks and gauge bosons, which have mass $m \sim g \sqrt{\xi}$. Instead at strong coupling, $\mathcal{N}=2$ SQCD is in the "instead-ofconfinement" phase [16,41], where perturbative states evolve into hadrons formed by the closed string states. All hadrons found from string theory of the non-Abelian vortex as closed string states turn out to be baryons and look like monopole "necklaces" [17].

To conclude this section, we note that our $4 \mathrm{D} \mathcal{N}=2$ SQCD has a Higgs branch formed by massless quarks which are in the bifundamental representation of the global 
group (2.2) and carry baryonic charge, see [14] for more details. This Higgs branch is hyper-Kählerian [42,43]; therefore, its metric cannot be modified by quantum corrections [43]. In particular, once the Higgs branch is present at weak coupling, we can continue it all the way into strong coupling. Thus, at strong coupling, in the "instead-of-confinement" phase besides stringy hadrons, we also have massless perturbative states: bifundamental quarks. It is argued in [17] that they play an important role in the stringy dynamics, see Sec. VII.

\section{B. World-sheet sigma model}

The presence of non-Abelian subgroup $\mathrm{SU}(N)_{C+F}$ in (2.2) is the reason for the formation of the non-Abelian vortex strings [1-4] in the 4D SQCD. The most important feature of these vortices is the presence of the so-called orientational zero modes. In this subsection, we are going to briefly review the sigma model emerging on the world sheet of the non-Abelian critical string supported in $\mathcal{N}=2$ SQCD with $N=2, N_{f}=4[10,14,15]$.

If $N_{f}=N$, the dynamics of the orientational zero modes of the non-Abelian vortex, which become orientational moduli fields on the world sheet, is described by the $2 \mathrm{D}$ $\mathcal{N}=(2,2)$ supersymmetric $\mathbb{C P}(N-1)$ model [8].

Upon adding extra quark flavors, non-Abelian vortices become semilocal. They acquire so-called size moduli [44]. In particular, for the non-Abelian semilocal vortex at hand with $N_{f}=4$, in addition to the orientational zero modes $n^{P}$ $(P=1,2)$, there are size moduli which we denote as $\rho^{K}$ $(K=1,2)$ [1,4,44-47].

The target space of the resulting weighted $\mathbb{C P}$ sigma model $[\mathbb{W} \mathbb{C P}(2,2)$ for short] is a symplectic quotient $\mathbb{C}^{4} / / \mathrm{U}(1)$, or in other words, the $\mathrm{U}(1) D$-term condition

$$
\left|n^{P}\right|^{2}-\left|\rho^{K}\right|^{2}=\beta
$$

plus U(1) gauge invariance. This quotient has complex dimension three.

Apart from these internal modes, the string under consideration has of course the usual translational modes corresponding to the $\mathbb{R}^{4}$ space that the string lives in. In total, the number of real bosonic degrees of freedom in this model is ten (four translational plus six internal). These degrees of freedom form a 10D space needed for superstring to be critical.

Since non-Abelian vortex string is $1 / 2 \mathrm{BPS}$, it preserves $\mathcal{N}=(2,2)$ in the world-sheet sigma model necessary for the $\mathcal{N}=2$ space-time supersymmetry $[48,49]$. Moreover, as was shown in [14], the string theory of the non-Abelian critical vortex is of type IIA.

The world-sheet coupling constant $\beta$ in (2.3) is related to the bulk gauge coupling constant $g^{2}$. At weak coupling, the relation is given by [8]

$$
\beta \approx \frac{4 \pi}{g^{2}}
$$

Note that the first (and the only) coefficient of the beta function is the same for the 4D SQCD and the worldsheet model. Both vanish at $N_{f}=2 N$. This ensures that our world-sheet theory is conformal. Therefore, its target space is Ricci flat and [being Kahler due to $\mathcal{N}=(2,2)$ supersymmetry] represents a noncompact Calabi-Yau manifold, namely, the conifold $Y_{6}$, see Ref. [13] for a review.

The global symmetry of the world-sheet sigma model is

$$
\mathrm{SU}(2) \times \mathrm{SU}(2) \times \mathrm{U}(1),
$$

i.e., exactly the same as the unbroken global group in the 4D theory, cf. (2.2), at $N=2$ and $N_{f}=4$. The fields $n$ and $\rho$ transform in the following representations:

$$
n:(\mathbf{2}, 0,0), \quad \rho:(0, \mathbf{2}, 1) .
$$

\section{Thin string regime}

The coupling constant of 4D SQCD can be complexified,

$$
\tau \equiv i \frac{4 \pi}{g^{2}}+\frac{\theta_{4 D}}{2 \pi},
$$

where $\theta_{4 D}$ is the $4 \mathrm{D} \theta$ angle. Note that the $\mathrm{SU}(N)$ version of the 4D SQCD at hand possesses a strong-weak coupling $(S)$ duality, namely, $\tau \rightarrow-\frac{1}{\tau}[43,50]$. There is also a symmetry with respect to the shift $\theta_{4 D} \rightarrow \theta_{4 D}+2 \pi$ ( $T$ duality).

The 2D coupling constant $\beta$ can be naturally complexified too if we include the $2 \mathrm{D} \theta$ term,

$$
\beta \rightarrow \beta+i \frac{\theta_{2 D}}{2 \pi} .
$$

The exact relation between the complexified 4D and 2D couplings was derived in Ref. [16],

$$
e^{-2 \pi \beta}=\lambda(2 \tau+1)
$$

where $\lambda$ is the elliptic modular lambda function. It can be expressed in terms of standard Jacobi $\theta$ functions [Ref. [51], p. 108] as

$$
\lambda(\tau)=\frac{\theta_{1}^{4}(\tau)}{\theta_{3}^{4}(\tau)} .
$$

Equation (2.9) generalizes the quasiclassical relation (2.4). It was derived using $2 \mathrm{D}-4 \mathrm{D}$ correspondence, namely, the match of the BPS spectra of the 4D theory at $\xi=0$ and the 
world-sheet theory on the non-Abelian string $[3,4,52,53]$. Note that our result (2.9) differs from that obtained in [54] by the shift of $\theta_{4 D}$ by $\pi$.

According to the hypothesis formulated in [10], our critical non-Abelian string becomes infinitely thin at strong coupling at the critical point $\tau_{c}$. Moreover, in [14], it was conjectured that $\tau_{c}$ corresponds to $\beta=0$ in the world-sheet theory via relation (2.9). The vortex transverse size is given by $1 / m$, where $m$ is the mass of $4 \mathrm{D}$ SQCD perturbative states. Thus, we assume that the mass of the 4D perturbative states $m \rightarrow \infty$ at $\beta=0$, which corresponds to $\tau=\tau_{c}$ in 4D SQCD.

At the point $\beta=0$, the non-Abelian string becomes infinitely thin, and higher derivative terms can be neglected. Also, the world-sheet theory of the non-Abelian string reduces to the $\mathbb{W} \mathbb{C P}(2,2)$ model. The point $\beta=0$ is a natural choice because at this point we have a regime change in the 2D sigma model. This is the point where the resolved conifold defined by the $D$ term constraint (2.3) develops a conical singularity [13]. Recently, it was shown in [16] that the point $\tau_{c}$, where our non-Abelian vortex string becomes thin, is $\tau_{c}=1$, which corresponds to $\beta=0$ via (2.9). ${ }^{4}$ This point is self-dual under a combination of $S$ and $T$ dualities.

When the parameter $\beta$ vanishes, the conifold singularity can be smoothened by a complex structure deformation. It was shown in [14] that the complex parameter $b$ of this deformation corresponds to a massless particle: a baryon with the baryon charge $Q_{B}=2$. This baryon can be represented as a "necklace" of four monopoles [16].

\section{NONCRITICAL $c=1$ STRING}

As was mentioned in Sec. I, the spectrum of massive string modes on the conifold was found in [17] (see also [18]) using an alternative formulation in terms of the $c=1$ string theory. This theory contains the Liouville field and a compact scalar at the self-dual radius and is argued to be equivalent to the string theory on the conifold in the so called double scaling limit [20-22]. In this section, we briefly review this theory and spectrum of physical string states obtained in [17].

The authors of [21,22] considered the above alternative formulation as a kind of AdS/CFT-type duality. ${ }^{5}$ Here, we follow the logic of Ref. [20] ${ }^{6}$ and consider it as an equivalence of two string theories: the critical string on the conifold and noncritical $c=1$ string with Liouville field, see also discussion in Sec. IV for more details.

\footnotetext{
${ }^{4}$ This corrects the value $\tau=i$ suggested earlier in [14,15].

${ }^{5}$ Below in this paper, we clarify the relation of our approach to AdS/CFT-type holography.

${ }^{6}$ In this paper, the equivalence was shown for topological versions of two string theories.
}

\section{A. Liouville theory}

Relevant noncritical $c=1$ string theory is formulated on the target space

$$
\mathbb{R}^{4} \times \mathbb{R}_{\phi} \times S^{1}
$$

where $\mathbb{R}_{\phi}$ is a real line associated with the Liouville field $\phi$, and the theory has a linear in $\phi$ dilaton, such that string coupling is given by

$$
g_{s}=e^{-\frac{Q}{2} \phi} .
$$

In [21], the authors proposed a more general equivalence between the critical string on noncompact Calabi-Yau spaces with isolated singularity on the one hand and noncritical $c=1$ string with the additional GinzburgLandau $\mathcal{N}=2$ superconformal system on the other hand. In our conifold case, this extra Ginzburg-Landau factor is absent from (3.1), see Ref. [22].

The bosonic stress tensor of the $c=1$ matter coupled to 2D gravity is given by [cf. the linear dilaton (3.2)],

$$
T_{--}=-\frac{1}{2}\left[\left(\partial_{z} \phi\right)^{2}+Q \partial_{z}^{2} \phi+\left(\partial_{z} Y\right)^{2}\right] .
$$

The compact scalar $Y$ represents $c=1$ matter and satisfies the periodicity condition

$$
Y \sim Y+2 \pi Q
$$

The scalars are normalized so that their propagators are

$$
\langle\phi(z), \phi(0)\rangle=-\log z \bar{z}, \quad\langle Y(z), Y(0)\rangle=-\log z \bar{z} .
$$

The central charge of the supersymmetric version of this $c=1$ theory is

$$
c_{\phi+Y}^{\mathrm{SUSY}}=3+3 Q^{2} .
$$

In order for the string on (3.1) to be critical, this central charge should be equal to 9 . This gives

$$
Q=\sqrt{2} .
$$

Deformation of the conifold (complex structure deformation) mentioned above translates into adding the Liouville interaction to the world-sheet sigma model [21],

$$
\delta L=b \int d^{2} \theta e^{-\frac{\phi+i Y}{Q}} .
$$

Here, $b$ is the deformation parameter. The conifold singularity at $b=0$ corresponds to the string coupling constant becoming infinitely large at $\phi \rightarrow-\infty$, see (3.2). At $b \neq 0$, 
the Liouville interaction regularizes the behavior of the string coupling preventing the string from propagating to the region of large negative $\phi$.

The mirror description of the Liouville $c=1$ noncritical string theory [23] is in terms of 2D black hole [24], which is the $\operatorname{SL}(2, \mathbb{R}) / \mathrm{U}(1)$ coset WZNW theory $[20,21,25,26]$ at the level

$$
k=\frac{2}{Q^{2}} .
$$

In the case of the conifold $(Q=\sqrt{2})$ this gives

$$
k=1 \text {, }
$$

where $k$ is the total level of the Kaĉ-Moody algebra in the supersymmetric version (the level of the bosonic part of the algebra is then $k_{b}=k+2=3$ ). The target space of this theory has the form of a semi-infinite cigar; the field $\phi$ associated with the motion along the cigar cannot take large negative values due to semi-infinite geometry.

In this description, the string coupling constant is largest at the tip of the cigar, $g_{s} \sim 1 / b$. We assume that parameter $b$ is large so the string coupling at the tip of the cigar is small, and the string perturbation theory becomes reliable. In particular, we can use the tree-level approximation to obtain the spring spectrum. Note also that, as we already mentioned in the Introduction, the $\mathrm{SL}(2, \mathbb{R}) / \mathrm{U}(1)$ WZNW model is exactly solvable, so we do not need to keep the level of the Kaĉ-Moody algebra $k$ large and can go to the strong coupling at $k=1$, see (3.10).

\section{B. Vertex operators}

Vertex operators for the string theory on (3.1) are constructed in [21], see also [22,25]. Primaries of the $c=$ 1 part for large positive $\phi$ (where the target space becomes a cylinder $\mathbb{R}_{\phi} \times S^{1}$ ) take the form

$V_{j ; m_{L}, m_{R}} \approx \exp \left\{\left(\sqrt{2} j \phi+i \sqrt{2}\left(m_{L} Y_{L}+m_{R} Y_{R}\right)\right)\right\}$,

where we split $Y$ into left- and right-moving parts. For the self-dual radius (3.7) (or $k=1$ ), the parameter $2 m$ in Eq. (3.11) is the integer. For the left-moving sector, $2 m_{L} \equiv$ $2 m$ is the total momentum plus the winding number along the compact dimension $Y$. For the right-moving sector, we introduce $2 m_{R}$ which is the momentum minus the winding number. For our case, the type IIA string $m_{R}=-m$, while for the type IIB string $m_{R}=m$ [18].

The primary operator (3.11) is related to the wave function over "extra dimensions" as follows:

$$
V_{j ; m_{L}, m_{R}}=g_{s} \Psi_{j ; m_{L}, m_{R}}(\phi, Y) .
$$

The string coupling (3.2) depends on $\phi$. Thus,

$$
\Psi_{j ; m_{L}, m_{R}}(\phi, Y) \sim e^{\sqrt{2}\left(j+\frac{1}{2}\right) \phi+i \sqrt{2}\left(m_{L} Y_{L}+m_{R} Y_{R}\right)} .
$$

We look for string states with normalizable wave functions over the "extra dimensions" which we interpret as hadrons in $4 \mathrm{D} \mathcal{N}=2 \mathrm{SQCD}$. The condition for the string states to have normalizable wave functions reduces to

$$
j \leq-\frac{1}{2} \text {. }
$$

The scaling dimension of the primary operator (3.11) is

$\Delta_{j, m}=\left.\frac{1}{k}\left\{m^{2}-j(j+1)\right\}\right|_{k=1}=m^{2}-j(j+1)$.

We include the case $j=-\frac{1}{2}$ which is at the borderline between normalizable and non-normalizable states. In Ref. [17], it is shown that $j=-\frac{1}{2}$ corresponds to the norm logarithmically divergent in the infrared in terms of the radial coordinate of the conifold. In particular, the massless baryon $b$ belongs to states with $j=-\frac{1}{2}$, see the next subsection.

Unitarity implies that the conformal dimension (3.14) should be positive,

$$
\Delta_{j, m}>0
$$

Moreover, to ensure that conformal dimensions of left- and right-moving parts of the vertex operator (3.11) are the same, we impose that $m_{R}= \pm m_{L}$.

The spectrum of the allowed values of $j$ and $m$ in (3.11) was exactly determined by using the Kaĉ-Moody algebra for the coset $\mathrm{SL}(2, R) / \mathrm{U}(1)$ in [25,27-30], see Ref. [55] for a review. Both discrete and continuous representations were found. Parameters $j$ and $m$ determine the global quadratic Casimir operator and the projection of the spin on the third axis,

$$
J^{2}|j, m\rangle=-j(j+1)|j, m\rangle, \quad J^{3}|j, m\rangle=m|j, m\rangle,
$$

where $J^{a}(a=1,2,3)$ are the global $\operatorname{SL}(2, R)$ currents.

We have

(i) Discrete representations with

$$
j=-\frac{1}{2},-1,-\frac{3}{2}, \ldots, \quad m= \pm\{j, j-1, j-2, \ldots\} .
$$

(ii) Principal continuous representations with

$j=-\frac{1}{2}+i s, \quad m=$ integer $\quad$ or $\quad m=$ half-integer,

where $s$ is a real parameter. 
(iii) Exceptional continuous representations with

$$
-\frac{1}{2} \leq j<0, \quad m=\text { integer. }
$$

We see that discrete representations include the normalizable states localized near the tip of the cigar, while the continuous representations contain non-normalizable states, ${ }^{7}$ see (3.13). This nicely matches our qualitative expectations.

Discrete representations contain states with negative norms. To exclude the ghost states, a restriction for spin $j$ is imposed [27-30,55],

$$
-\frac{k+2}{2}<j<0
$$

Thus, for our value $k=1$, we are left with only two allowed values of $j$,

$$
j=-\frac{1}{2}, \quad m= \pm\left\{\frac{1}{2}, \frac{3}{2}, \ldots\right\},
$$

and

$$
j=-1, \quad m= \pm\{1,2, \ldots\} .
$$

\section{Scalar and spin-2 states}

Four-dimensional spin- 0 and spin- 2 states were found in [17] using vertex operators (3.11). The 4D scalar vertices $V^{S}$ in the $(-1,-1)$ picture have the form [21]

$$
V_{j ; m,-m}^{S}=e^{-\varphi_{L}-\varphi_{R}} e^{i p_{\mu} x^{\mu}} V_{j ; m,-m},
$$

where superscript $S$ stands for scalar, $\varphi_{L, R}$ represent bosonised ghosts in the left- and right-moving sectors, while $p_{\mu}$ is the $4 \mathrm{D}$ momentum of the string state.

The condition for the state (3.23) to be physical is

$$
\frac{1}{2}+\frac{p_{\mu} p^{\mu}}{8 \pi T}+m^{2}-j(j+1)=1,
$$

where (3.14) was used. The first term on the lhs comes from ghosts [recall that the conformal dimension of the ghost operator $e^{q \varphi}$ is equal to $-\left(q+q^{2} / 2\right)$ in the picture $q$ ].

The GSO projection restricts the integer $2 m$ for the operator in $(3.23)$ to be odd $[21,56]$,

$$
m=\frac{1}{2}+\mathbb{Z}
$$

\footnotetext{
${ }^{7}$ We discuss the case $j=-\frac{1}{2}$ which is on the borderline between normalizable and non-normalizable states in the next subsection.
}

For half-integer $m$, we have only one possibility $j=-\frac{1}{2}$, see (3.21). This determines the masses of the 4D scalars,

$$
\frac{\left(M_{m}^{S}\right)^{2}}{8 \pi T}=-\frac{p_{\mu} p^{\mu}}{8 \pi T}=m^{2}-\frac{1}{4},
$$

where the Minkowski 4D metric of the signature $(-1,1,1,1)$ is used. It is shown in [17] that the state with $m= \pm 1 / 2$ is the massless baryon $b$, associated with deformations of the conifold complex structure [14]. It consists of four monopoles in a "necklace" formed by a closed string [16]. Higher states with $m= \pm(3 / 2,5 / 2, \ldots)$ are massive 4D scalars.

At the next level, we consider 4D spin-2 states. The corresponding vertex operators are given by

$$
\left(V_{j ; m,-m}\left(p_{\mu}\right)\right)^{\mathrm{spin}-2}=\xi_{\mu \nu} \psi_{L}^{\mu} \psi_{R}^{\nu} e^{-\varphi_{L}-\varphi_{R}} e^{i p_{\mu} x^{\mu}} V_{j ; m,-m},
$$

where $\psi_{L, R}^{\mu}$ are the world-sheet superpartners to $4 \mathrm{D}$ coordinates $x^{\mu}$, while $\xi_{\mu \nu}$ is the polarization tensor. For these states to be physical, one should impose a condition

$$
\frac{p_{\mu} p^{\mu}}{8 \pi T}+m^{2}-j(j+1)=0 .
$$

The GSO projection selects now $2 m$ to be even, $|m|=$ $0,1,2, \ldots[21]$; thus, we are left with only one allowed value of $j, j=-1$ in Eq. (3.22). Moreover, the value $m=0$ is excluded. This leads to the following expression for the masses of spin-2 states:

$$
\left(M_{m}^{\text {spin-2 }}\right)^{2}=8 \pi T m^{2}, \quad|m|=1,2, \ldots .
$$

All spin-2 states are massive, and no massless 4D graviton appears in our theory. It matches the fact that our $4 \mathrm{D} \mathcal{N}=2$ QCD is defined in flat space without gravity.

The momentum $m$ in the compact dimension is related to the baryonic charge. It was shown in $[17,18]$ that the baryon charge of the vertex operators (3.23) and (3.27) is given by

$$
Q_{B}=4 m
$$

All closed string states are baryons.

\section{SOLITONIC STRING-GAUGE DUALITY VERSUS HOLOGRAPHY}

As we already mentioned in the Introduction, a version of the string-gauge duality for $4 \mathrm{D} \mathcal{N}=2 \mathrm{SQCD}$ with $N_{f}=$ $2 N=4$ was proposed in [10]; at weak coupling, this theory is in the Higgs phase and can be described in terms of (s) quarks and Higgsed gauge bosons, while at strong coupling hadrons of this theory can be understood as closed string states formed by the non-Abelian vortex string. This duality was further explored by studying the string theory for the critical non-Abelian vortex in $[14,15,17]$. 
We call this duality a solitonic string-gauge duality and would like to compare it with AdS/CFT-type holography. Of course, there is a conceptual difference. In our approach $10 \mathrm{D}$ space is an artificial construction formed by 4D "real" space where SQCD lives and six-dimensional conifold associated with orientational and size moduli of the nonAbelian vortex. Instead, in AdS/CFT correspondence, the fundamental superstring propagates in the $10 \mathrm{D}$ space from the very beginning.

Also the scales of the string tension are dramatically different in two approaches. The scale of the squire root of the fundamental string tension is determined roughly speaking by the Plank scale, while for the solitonic nonAbelian vortex $\sqrt{T}$ is fixed at the SQCD scale set by the FI parameter, see (2.1).

However, we can take a more pragmatic point of view "forgetting" for a minute where the 10D space comes from and think of our solitonic string-gauge duality as a duality which associates a given 4D SQCD with a string theory on the conifold. Then, we can summarize the main distinctions of our approach as follows:

(i) AdS/CFT correspondence is typically based on the presence of $N_{b}$ parallel $D$-branes, see, for example, [37] for a review. Moreover, the validity of the gravity approximation requires a large $N_{b}$ limit. In our string theory on the conifold, there are no branes. The validity of our approximation is based on the large value of the parameter of the conifold complex structure deformation $b$. Large $b$ ensures the validity of the gravity approximation because the curvature of the conifold remains everywhere small, see, for example, [57]. Moreover, as we already mentioned, large $b$ ensures small $g_{s}$ in the string theory formulated on the cigar.

(ii) AdS/CFT correspondence assumes holography. Off-shell correlation functions on the field theory side correspond to string theory correlation functions on the "boundary," infinitely far away from the branes. Instead, in our solitonic string-gauge duality, all nontrivial "real" physics should be localized exclusively near the tip of the cigar. We consider only normalizable vertex operators with $j \leq-\frac{1}{2}$ [see (3.13)] and identify them with hadrons of 4D SQCD.

The first distinction above suggests that we can think of our solitonic string-gauge duality as of $N_{b}=0$ limit of AdS/CFT correspondence. The simplest example is the Klebanov-Witten's construction [58] of $N_{b}$ D3-branes filling the $\mathbb{R}^{4}$ space near the conifold singularity in a type IIB superstring. The presence of the five-form flux sourced by branes makes a direct product of $\mathbb{R}^{4}$ and $Y_{6}$ a warped product with anti-de Sitter $\left(\mathrm{AdS}_{5}\right)$ geometry. In the limit $N_{b}=0$, the warped factor disappears, and we get our background $\mathbb{R}^{4} \times Y_{6}$.

However, the second distinction seems to be a crucial one. To clarify this issue, below in this paper, we compare our theory with the most close example of the AdS/CFT holography: nongravitational six-dimensional LST on the world volume of $k N S 5$-branes [21,38,39]. This theory is holographically dual to the noncritical string theory on $\mathbb{R}^{6} \times \mathrm{SL}(2, \mathbb{R})_{k} / \mathrm{U}(1) \times \mathrm{SU}(2)_{k} / \mathrm{U}(1)$, where $k$ is the level of the Kac-Moody algebra of WZNW model. ${ }^{8}$ The duality ensures that off-shell correlation functions in LST corresponds to correlation functions of non-normalizable vertex operators on the cigar, see Ref. [19] for a review.

Below, we test this type of holography for the string theory of our non-Abelian vortex. In particular, we test the LSZ relation (1.1) found in [39] for the theory on NS5branes. First, we find the poles of two-point correlation functions of non-normalizable vertex operators and compare results to the hadron spectrum found previously [17,18], see Eqs. (3.26) and (3.29). The poles must directly correspond to hadron masses. Second, we consider the correlation functions of normalizable vs. non-normalizable operators and see how they are related to each other.

To conclude this section, we note that in $[21,22]$ the above holographic duality for LST on NS5-branes was generalized to the correspondence between the critical string on noncompact Calabi-Yau spaces with isolated singularity in the double scaling limit on the one hand and noncritical $c=1$ string with the additional GinzburgLandau $\mathcal{N}=2$ superconformal system on the other hand. The equivalence of the critical string on the conifold and noncritical $c=1$ string we use here is a particular case of this correspondence. However, this correspondence looks more like equivalence of two closed string theories rather then duality between open and closed string descriptions typical for AdS/CFT-type holography. Therefore, as we already mentioned, we follow the logic of Ref. [20] and consider this correspondence as an equivalence of two string theories rather than the AdS/CFT-type duality. One of the important directions of future work is to demonstrate this equivalence more directly.

\section{TWO-POINT CORRELATION FUNCTIONS AND (NON-)NORMALIZABLE OPERATORS}

\section{A. Reflection property}

As we already mentioned, $\operatorname{SL}(2, \mathbb{R}) / U(1)$ primary fields were constructed in [21], see also Refs. [22,25] and [33,34]. Primaries of the $c=1$ part can be expanded at $\phi \rightarrow \infty$ where the target space becomes a cylinder $\mathbb{R}_{\phi} \times S^{1}$. In (3.11), we presented the leading at large $\phi$ term for values of $j$ associated with discrete series (3.17). For generic $j$, the asymptotic expansion can be written as follows ${ }^{9}$ [34]:

\footnotetext{
${ }^{8}$ In our string theory, the last factor [compact SU(2)/U(1) CFT] is absent.

${ }^{9}$ Similar formulas have appeared also in [39], but in this paper, we use the normalization of [34], see Eqs. (13)-(14) and (16)(20) in the latter paper. Also, our notation for $m_{R}, m_{L}$ modes is related to the notation $m, \bar{m}$ of $[21,39]$ as $m=m_{L}, \bar{m}=-m_{R}$.
} 


$$
\begin{aligned}
V_{j, m_{L}, m_{R}}= & e^{i Q\left(m_{L} Y_{L}+m_{R} Y_{R}\right)}\left[e^{Q j \phi}\right. \\
& \left.+R\left(j, m_{L}, m_{R} ; k\right) e^{-Q(j+1) \phi}+\cdots\right],
\end{aligned}
$$

where the ellipses stand for terms that are subleading at large positive $\phi$. The background charge $Q$ is related to the level $k$ via (3.9) as $k=2 / Q^{2}$. We keep $k$ arbitrary and put it to the value $k=1$ [see Eq. (3.10)] at the last step. The so-called reflection coefficient $R\left(j, m_{L}, m_{R} ; k\right)$ is given by Ref. [39]

$$
\begin{aligned}
& R\left(j, m_{L}, m_{R} ; k\right) \\
& =\left[\frac{1}{\pi} \frac{\Gamma\left(1+\frac{1}{k}\right)}{\Gamma\left(1-\frac{1}{k}\right)}\right]^{2 j+1} \\
& \quad \times \frac{\Gamma\left(1-\frac{2 j+1}{k}\right) \Gamma\left(m_{L}+j+1\right) \Gamma\left(m_{R}+j+1\right) \Gamma(-2 j-1)}{\Gamma\left(1+\frac{2 j+1}{k}\right) \Gamma\left(m_{L}-j\right) \Gamma\left(m_{R}-j\right) \Gamma(2 j+1)} .
\end{aligned}
$$

Physically, the presence of the two exponentials written in the expansion (5.1) represents a reflection from the tip of the cigar. Note that these two exponentials have the same conformal dimension, see Eq. (3.14). For our case of a type IIA string, we take $m_{L}=-m_{R}=m$.

The reflection coefficient (5.2) is defined up to an arbitrary factor of the form $(A)^{2 j+1}$, where $A$ is a constant. It can be absorbed into a redefinition of the operators

$$
V_{j, m_{L}, m_{R}} \mapsto(A)^{j} V_{j, m_{L}, m_{R}}
$$

see Ref. [39]. We use the normalization of [33,34,39].

The primaries under consideration obey the so-called reflection property [39],

$$
V_{j ; m_{L}, m_{R}}=R\left(j, m_{L}, m_{R} ; k\right) V_{-j-1 ; m_{L}, m_{R}} .
$$

Note a useful relation

$$
R\left(j, m_{L}, m_{R} ; k\right) \cdot R\left(-j-1, m_{L}, m_{R} ; k\right)=1 .
$$

The reflection property (5.4) can be easily checked on the level of expansion (5.1). We have

$$
\begin{aligned}
V_{j, m_{L}, m_{R}} \approx & e^{i Q\left(m_{L} Y_{L}+m_{R} Y_{R}\right)}\left[e^{Q j \phi}+R\left(j, m_{L}, m_{R} ; k\right) e^{-Q(j+1) \phi}\right] \\
= & R\left(j, m_{L}, m_{R} ; k\right) e^{i Q\left(m_{L} Y_{L}+m_{R} Y_{R}\right)} \\
& \times\left[R\left(-j-1, m_{L}, m_{R} ; k\right) e^{Q j \phi}+e^{-Q(j+1) \phi}\right] \\
\approx & R\left(j, m_{L}, m_{R} ; k\right) V_{-j-1 ; m_{L}, m_{R}}
\end{aligned}
$$

where we use Eq. (5.5).
For generic values of $j$, one exponential in Eq. (5.1) is normalizable, while the other one is non-normalizable, see Eq. (3.13), which stays intact for generic values of $k$. However, at special values of $j, m_{L}, m_{R}$, these operators can become normalizable. This can happen in one of the following cases:

(1) If $j<-1 / 2$ and the reflection coefficient $R\left(j, m_{L}, m_{R} ; k\right)$ vanishes.

(2) If $j>-1 / 2$ and the reflection coefficient $R\left(j, m_{L}, m_{R} ; k\right)$ has a pole.

(3) If $j=-1 / 2$.

To see this, consider an operator $V_{j ; m_{L}, m_{R}}$ with $j<-1 / 2$. When $j$ and $m_{L}, m_{R}$ approach the values of the discrete series (3.17), the reflection coefficient vanishes. Technically it happens because some gamma functions in Eq. (5.2) develop poles; for more details. see below. Using the expansion (5.1). we find

$$
\begin{aligned}
V_{j ; m_{L}, m_{R}} \sim & e^{i Q\left(m_{L} Y_{L}+m_{R} Y_{R}\right)} \\
& \times(e^{Q j \phi}+\underbrace{R\left(j, m_{L}, m_{R} ; k\right)}_{\rightarrow 0} e^{-Q(j+1) \phi}) \rightarrow \\
\rightarrow & e^{Q j \phi+i Q\left(m_{L} Y_{L}+m_{R} Y_{R}\right)},
\end{aligned}
$$

which is the leading term in the expansion of a normalizable operator.

For an operator $V_{\tilde{j}}$ with $\tilde{j}=-1-j>-1 / 2$. we have similarly

$$
\begin{aligned}
V_{\tilde{j} ; m_{L}, m_{R}} & \sim e^{i Q\left(m_{L} Y_{L}+m_{R} Y_{R}\right)}(e^{Q \tilde{j} \phi}+\underbrace{R\left(\tilde{j}, m_{L}, m_{R} ; k\right)}_{\text {has a pole }} e^{Q j \phi}) \rightarrow \\
& \rightarrow R\left(\tilde{j}, m_{L}, m_{R} ; k\right) e^{Q j \phi+i Q\left(m_{L} Y_{L}+m_{R} Y_{R}\right)},
\end{aligned}
$$

[cf. (5.5)]. This expression contains a pole. The residue at this pole is again a normalizable operator (see below for more details).

As for $j=-\frac{1}{2}$, it is a borderline case when the two exponentials in Eq. (5.1) are the same. As we already mentioned, it is a state with the norm logarithmically divergent in the infrared. As was argued in [17], this state should be included into the physical spectrum.

Thus, we can have normalizable as well as non-normalizable operators.

In the following, we are interested in correlation functions of operators corresponding to hadronic states of $4 \mathrm{D}$ SQCD. Strictly speaking, these are given by "dressed" vertex operators (3.23) and (3.27), and apart from the $\mathrm{SL}(2, R) / \mathrm{U}(1)$ part, they include ghost and $\mathbb{R}^{4}$ factors. However these "extra" factors contribute to the correlation functions trivially. Below, we focus on the "internal" $(\phi, Y)$-dependent $\mathrm{SL}(2, R) / \mathrm{U}(1)$ part in the correlation functions. The latter will contain all the relevant pole structures, and only this $(\phi, Y)$-dependent part is relevant 
for studying normalizable and non-normalizable operators in the sense discussed above.

\section{B. Poles of the two-point function and holography}

AdS/CFT-type holography discussed in Sec. IV relates correlation functions of non-normalizable operators with $j>-1 / 2$ to correlation functions of normalizable operators with $j<-1 / 2$, see Eqs. (1.1) and (5.8). The reflection property (5.4) and the expansion (5.1) are the main technical ingredients necessary for understanding this relation.

The two-point correlation function of the primary operators was computed in [21] (see also Refs. [33,34] and $[39,59])$. The exact formula reads ${ }^{10}$

$$
\left\langle V_{j_{1} ; m_{L}, m_{R}} V_{j_{2} ;-m_{L},-m_{R}}\right\rangle=R\left(j, m_{L}, m_{R} ; k\right) \delta\left(j_{1}-j_{2}\right),
$$

with the quantity $R$ (the reflection coefficient) given by (5.2). A quasiclassical justification of this formula (5.9) is presented in Appendix C. Note that here and below we suppress the standard dependence of the world-sheet coordinates.

First, we note that since the two-point correlation function (5.9) equals the reflection coefficient (5.2), the poles of the correlation function exactly correspond to the values of $j, m_{L}, m_{R}$ for which the operators inside the correlation function become normalizable, see Sec. VA. Second, when we consider correlation functions of normalizable operators with $j \leq-1 / 2$, at special values of $j, m_{L}, m_{R}$, they turn out to be finite, as we see below.

The non-normalizable operator with $j>-1 / 2$ at special values of $j, m$ reduces to a normalizable operator times a reflection coefficient with a pole. This ensures the relation (1.1) found in [39]. Therefore, we can expect that the twopoint correlation function of two such operators has a pole.

As we explain in Sec. IV, we can interpret this in the spirit of AdS/CFT-type holography which assumes that off mass-shell correlation functions in the 4D field theory are given by string correlation functions of non-normalizable operators. Poles are associated with propagation of a physical state.

Let us reiterate. Correlation function $\left\langle V_{\tilde{j}, m_{L}, m_{R}}, O_{1}\right.$, $\left.\ldots, O_{n}\right\rangle$ with a non-normalizable operator $V_{\tilde{j}, m_{L}, m_{R}}$ with $\tilde{j}>-1 / 2$ corresponds to a correlation function $\left\langle V_{j, m_{L}, m_{R}}\right.$, $\left.O_{1}, \ldots, O_{n}\right\rangle$ with a normalizable operator $V_{j, m_{L}, m_{R}}$ with $j<-1 / 2$ [see (5.8)]. Here $O_{1}, \ldots, O_{n}$ denote other vertex operators in a correlation function.

\footnotetext{
${ }^{10}$ In this paper, we mostly do not write the dependence on the world-sheet coordinates explicitly. Moreover, note the $\delta\left(j_{1}-j_{2}\right)$ factor is absent in $[39,59]$. The authors of those papers argued that this delta function cancels against the ghost zero mode that would normally make the two-point amplitude vanish in string theory. However according to recent work [60], the two-point string amplitudes are nonvanishing after all. Therefore, we should keep the delta function $\delta\left(j_{1}-j_{2}\right)$.
}

Specifically, $j$ and $\tilde{j}$ for these two operators are related as

$$
\underbrace{\tilde{j}}_{\text {non-norm }}=-1-\underbrace{j}_{\text {norm }} \text {. }
$$

As we already mentioned, these two vertex operators $V_{\tilde{j}, m_{L}, m_{R}}$ and $V_{j, m_{L}, m_{R}}$ have the same conformal dimension, and we expect that as $\left(j, m_{L}, m_{R}\right)$ approach values for the discrete spectrum the reflection coefficient develops a pole. Then, two vertex operators $V_{\tilde{j}, m_{L}, m_{R}}$ and $V_{j, m_{L}, m_{R}}$ satisfy the LSZ relation (1.1) which can be called "the holography relation". The pole of the propagator of a 4D physical state in this relation comes from the pole of the reflection coefficient with respect to $\left(j, m_{L}, m_{R}\right)$ via relations (3.24) or (3.28).

\section{Poles of the two-point function and discrete representations of $\operatorname{SL}(2, \mathbb{R}) / \mathbf{U}(\mathbf{1})$}

Now let us look more closely at pole structures of two-point correlation functions.

Poles of the correlation functions of operators living on the covering space of $\operatorname{SL}(2, \mathbb{R})$ were analyzed in $[39,59]$. Because of the cover, the values of $m=m_{L}$ in the vertex operators (5.1) are not quantized in this setting. Moreover, the $\operatorname{SL}(2, \mathbb{R})$-spin $j$ was not restricted to take discrete values in $[39,59]$.

In our setup, the field $Y$ in (5.1) in the WZNW formulation lives on a circle of radius $R_{s l}=\sqrt{2 k}^{11}$ It is a periodic variable. We do not consider the covering space. Instead, we require the primaries to be $2 \pi R_{s l}$ periodic with respect to the field $Y$. This immediately imposes a condition

$$
2 m \in \mathbb{Z},
$$

for arbitrary $k$. In the following, we always assume that (5.11) is true.

We are going to consider a two-point function (5.9) of two non-normalizable operators with $\tilde{j}=-1-j>-1 / 2$. We are interested in poles of this two-point function that depend on both $j$ and $m$ (in [39] they were called LSZ poles). It turns out that the values of $j, m$ corresponding to these poles exactly correspond to discrete representations of $\operatorname{SL}(2, \mathbb{R}) / \mathrm{U}(1)$ (3.17). Here, we show that the physical poles of the two-point correlation functions of non-normalizable operators give exactly the $j=-1$ series. The operators with $j=-1 / 2$ are on the borderline between normalizable and non-normalizable, and the corresponding two-point function turns out to be finite.

\footnotetext{
${ }^{11}$ Note that the radius of a circle in the Liouville formulation is given by (3.4), while the radius of a circle in the mirror WZNW formulation is given by $R_{s l}=\sqrt{2 k}$, see [21]. To simplify the notation, we use the same notation $Y$ for this periodic field.
} 
Note that one should be careful when analyzing potentially divergent quantities. From what has been said so far, we can deduce the following rule: when investigating the poles of $j, m, k$-dependent quantities (e.g., correlation functions), we have to take the limits in the following order:

(1) First, take $m$ to be a half-integer (5.11).

(2) Next, send $j$ to the desired value $j_{0}$. Holography relation (1.1) suggests that it corresponds to the discrete representation of $\mathrm{SL}(2, \mathbb{R}) / \mathrm{U}(1)$, e.g., $j \rightarrow-1 / 2$ or $j \rightarrow-1$, see Eqs. (3.21) and (3.22).

(3) Lastly, take the limit $k \rightarrow 1$.

This ensures that the LSZ "holography relation" (1.1) suggested in [39] can be written in our theory as follows:

$$
\left\langle V_{\tilde{j}, m_{L}, m_{R}}, O_{1}, \ldots, O_{n}\right\rangle \sim \frac{1}{j-j_{0}}\left\langle V_{j, m_{L}, m_{R}}, O_{1}, \ldots, O_{n}\right\rangle,
$$

near the pole at $j \rightarrow j_{0}$, where we fixed $m$ to be a (half) integer. Here, $\tilde{j}$ and $j$ are related via (5.10), while $j_{0}=-1$. Below, we check this relation for the two and three-point correlation functions in our theory.

We also check the holography relation (1.1) for the $j_{0}=-1 / 2$ channel and show that holography does not work in this case.

$$
\text { 1. } j=-1
$$

Let us consider the two-point correlation function (5.9) with two non-normalizable operators $V_{\tilde{j}, m_{L}, m_{R}}$ near $\tilde{j}=-1-j=0$. To investigate the pole structure, we can use a technique similar to the one outlined in [59] [in particular, see Eqs. (3.10)-(3.13) in that paper]. Let us take $m_{L}=-m_{R} \equiv m>0$. Using Eq. (5.9) and expanding the $\Gamma$ functions in (5.2), we can see that the two-point correlation function has a pole at $\tilde{j}=0$,

$$
\left\langle V_{\tilde{j} ; m,-m} V_{\tilde{j}^{\prime} ;-m, m}\right\rangle=R_{m} \frac{1}{\tilde{j}} \delta\left(\tilde{j}-\tilde{j}^{\prime}\right),
$$

with the residue given by (see Appendix D)

$$
R_{m}=\underset{\tilde{j}=0}{\operatorname{Res}} R(\tilde{j}, m,-m ; k)=\frac{m^{2}}{2 \pi} .
$$

This formula holds also for $m<0$. Recalling that $\tilde{j}=-1-j$, we see that the singularity actually appears at

$$
j=-1, \quad m= \pm\{1,2, \ldots\},
$$

and the two-point correlation function (5.13) can be rewritten as

$$
\left\langle V_{\tilde{j} ; m,-m} V_{\tilde{j}^{\prime} ;-m, m}\right\rangle=\frac{R_{m}}{-1-j} \delta\left(\tilde{j}-\tilde{j}^{\prime}\right)
$$

which precisely corresponds to a $j=-1$ discrete series of $\mathrm{SL}(2, R) / \mathrm{U}(1)$ representations (3.22). Note that the residue (5.14) vanishes at $m=0$ which directly corresponds to the fact that $m=0$ associated with the would-be massless 4D graviton is excluded from the series (3.22). Poles in (5.16) correspond to a part of massive physical states (3.29) found in [17].

Thus, we see that AdS/CFT-type holography works in our theory for $4 \mathrm{D}$ physical states with $j=-1$. Namely, these 4D SQCD states are seen as poles in correlation functions of non-normalizable vertex operators in the string theory in accordance with Eqs. (1.1) and (5.12).

Let us note that at $2 m \in \mathbb{Z}$, the two-point correlation function also has other poles at $\tilde{j}>0$ (corresponding to $j<-1$ ). Similar poles are called "bulk poles" in [39] because they come from the region of large $\phi$ rather then from states localized near the tip of the cigar (which are called LSZ poles), see also discussions in Refs. [21,61,62]. In our theory, these poles are not physical and should be ignored. Associated states with $j<-1$ break the lower bound in Eq. (3.20) and have negative norms.

Finally, let us make a note on the two-point function of the corresponding normalizable operators. According to Eq. (5.9),

$$
\left\langle V_{j=-1 ; m,-m} V_{j=-1 ;-m, m}\right\rangle=R(j=-1, m,-m ; k) \delta(0) .
$$

The reflection coefficient here vanishes, but this zero cancels against the delta function. The whole two-point function is finite.

\section{2. $j=-1 / 2$ normalizable}

Now consider series of states with $j=-1 / 2$, see Eq. (3.21). These states are on the borderline between normalizable and non-normalizable and saturate the socalled Seiberg bound [63]). As we already mentioned, they are logarithmically normalizable with respect to the conifold radial coordinate and were interpreted as physical states, see [17] and Sec. III B. Let us have a closer look at the two-point function of these states.

Let us set for a moment $j=-1 / 2-\epsilon, m_{L}=-m_{R} \equiv$ $m=1 / 2+\delta$. We have 


$$
\begin{aligned}
\left\langle V_{j ; m,-m} V_{j ;-m, m}\right\rangle & =R(j, m,-m ; k) \\
& =\left[\frac{1}{\pi} \frac{\Gamma\left(1+\frac{1}{k}\right)}{\Gamma\left(1-\frac{1}{k}\right)}\right]^{2 j+1} \frac{\Gamma\left(1-\frac{2 j+1}{k}\right) \Gamma(j+m+1) \Gamma(j-m+1) \Gamma(-2 j-1)}{\Gamma\left(1+\frac{2 j+1}{k}\right) \Gamma(m-j) \Gamma(-j-m) \Gamma(2 j+1)} \\
& =\left[\frac{1}{\pi} \frac{\Gamma\left(1+\frac{1}{k}\right)}{\Gamma\left(1-\frac{1}{k}\right)}\right]^{-2 \epsilon} \frac{\Gamma\left(1+\frac{2 \epsilon}{k}\right) \Gamma(1-\epsilon+\delta) \Gamma(-\epsilon-\delta) \Gamma(2 \epsilon)}{\Gamma\left(1-\frac{2 \epsilon}{k}\right) \Gamma(1+\delta+\epsilon) \Gamma(\epsilon-\delta) \Gamma(-2 \epsilon)} \\
& =\frac{\epsilon-\delta}{\epsilon+\delta}+\text { (nonsingular terms). }
\end{aligned}
$$

This analysis can be repeated for other values of $m, m \in$ $1 / 2+\mathbb{Z}$ with the same result. One can see that, generally speaking, this expression is ambiguous. However, by our prescription, we have to first set $m=1 / 2$ (i.e., $\delta=0$ ) and then take the limit $j \rightarrow-1 / 2$ (i.e., $\epsilon \rightarrow 0$ ). Finally, we take the limit $k \rightarrow 1$. Then, the expression for the two-point correlation function is well defined, and we have

$$
\left\langle V_{-1 / 2 ; m,-m} V_{-1 / 2 ;-m, m}\right\rangle \equiv \lim _{j \rightarrow-1 / 2}\left\langle V_{j ; m,-m} V_{j ;-m, m}\right\rangle=1
$$

The correlation function is finite. This confirms the interpretation of $j=-\frac{1}{2}$ states as physical states (logarithmically) localized near the tip of the cigar.

Finally, let us note that the factor $\delta\left(j_{1}-j_{2}\right)$ coming from Eq. (5.9) in fact does not enter the final expression for the two-point function for $j=-1 / 2$. For details, see Sec. VII.

\section{3. $j=-1 / 2$ non-normalizable}

Since the value $j=-1 / 2$ is invariant under the reflection (5.10), one may think that there is no corresponding non-normalizable operator. It turns out that this is not entirely true. We saw that for each operator $V_{j}$ with $j \neq$ $-1 / 2$ there is an operator $V_{\tilde{j}}, \tilde{j}=-j-1$ with the same conformal dimension. This is related to the fact that the Schrödinger equation in the corresponding quantum mechanical problem has two solutions. In the case of the operator $V_{j=-1 / 2}$, the second solution to the Schrödinger equation is the operator [63]

$$
\phi V_{\tilde{j}=-1 / 2, m,-m} \sim \phi e^{-\frac{Q}{2} \phi} e^{i Q\left(m Y_{L}-m Y_{R}\right)} .
$$

Therefore, we should take this operator into account.

The operator (5.20) is an example of a so-called logarithmic primary field [64] (see also [65,66] and references therein). Such operators were also considered to some extent in [56,67]. It is a primary field with the same conformal dimension as $V_{j=-1 / 2, m,-m} \sim$ $e^{-Q / 2 \phi} e^{i Q\left(m Y_{L}-m Y_{R}\right)}$. Indeed, consider its pairing with the energy-momentum tensor,

$$
\begin{aligned}
\left\langle\phi e^{Q j \phi} e^{i Q\left(m Y_{L}-m Y_{R}\right)}\left(z_{1}\right), T\left(z_{2}\right)\right\rangle & =\frac{1}{Q} \partial_{j}\left\langle e^{Q j \phi} e^{i Q\left(m Y_{L}-m Y_{R}\right)}\left(z_{1}\right), T\left(z_{2}\right)\right\rangle \\
& =\frac{1}{\left(z_{1}-z_{2}\right)^{2}} \frac{1}{Q} \partial_{j}\left(\Delta_{j, m} e^{Q j \phi} e^{i Q\left(m Y_{L}-m Y_{R}\right)}+\ldots\right) \\
& =\left.\frac{e^{i Q\left(m Y_{L}-m Y_{R}\right)}}{\left(z_{1}-z_{2}\right)^{2}}\left(-\frac{1}{Q} \frac{2 j+1}{k} e^{Q j \phi}+\Delta_{j, m} \phi e^{Q j \phi}\right)\right|_{j=-\frac{1}{2}}+\cdots \\
& =\frac{\Delta_{j, m}}{\left(z_{1}-z_{2}\right)^{2}} \phi e^{Q j \phi} e^{i Q\left(m Y_{L}-m Y_{R}\right)}+\ldots,
\end{aligned}
$$

where $z_{1}$ and $z_{2}$ are world-sheet coordinates. This shows that operator

$$
\phi V_{\tilde{j}=-1 / 2, m,-m} \sim \phi e^{-\frac{Q}{2} \phi} e^{i Q\left(m Y_{L}-m Y_{R}\right)}
$$

is a primary field with conformal dimension $\Delta_{j=-1 / 2, m}$, where $\Delta_{j, m}$ is defined in (3.14). The associated wave function is more divergent at large $\phi$ than the one for
$V_{j=-1 / 2}$; therefore, we consider the operator (5.20) as a non-normalizable "partner" of $V_{j=-1 / 2}$.

The corresponding two-point correlation function turns out to have a double pole (see Appendix B 8 for the details),

$$
\left\langle\phi V_{\tilde{j}_{1}=-1 / 2, m,-m} \phi V_{\tilde{j}_{2}=-1 / 2,-m, m}\right\rangle \sim \frac{1}{\left(\tilde{j}_{1}+\frac{1}{2}\right)^{2}} \delta\left(\tilde{j}_{1}-\tilde{j}_{2}\right) .
$$


The double pole is exactly what one would expect for the correlation function (5.22) from LSZ. From Eq. (3.24), we see that the linear in $(\tilde{j}+1 / 2)$ term vanishes at $\tilde{j} \rightarrow-1 / 2$ and we have

$$
p_{\mu}^{2}+M^{2} \sim\left(\tilde{j}+\frac{1}{2}\right)^{2} \text { near } \tilde{j} \rightarrow-\frac{1}{2} .
$$

Therefore, finally we get the LSZ formula,

$$
\begin{aligned}
& \left\langle\phi V_{\tilde{j}_{1}=-1 / 2, m,-m} \phi V_{\tilde{j}_{2}=-1 / 2,-m, m}\right\rangle \\
& \quad \sim \frac{1}{p_{\mu}^{2}+M^{2}}\left\langle V_{j=-1 / 2, m,-m} V_{j=-1 / 2,-m, m}\right\rangle .
\end{aligned}
$$

We conclude that the LSZ pole is present for the nonnormalizable $j=-1 / 2$ operator (5.20), so the holography works in this channel for the two-point function. Below, we see that it does not work for the three-point function of such operators.

\section{THREE-POINT CORRELATION FUNCTIONS}

In this section, we consider three-point correlation functions of operators $V_{j, m_{L}, m_{R}}$ with $m_{L}=-m_{R} \equiv m$. The physical states at our disposal correspond to normalizable operators with $j=-1,-1 / 2$ or by (5.10) to $\tilde{j}=0,-1 / 2$. The values of $m$ of the three correlation functions have to sum up to zero,

$$
m_{1}+m_{2}+m_{3}=0 .
$$

This corresponds to baryon charge conservation in the 4D SQCD, see (3.30). Winding number conservation in threepoint functions was also discussed in [62].

Therefore, we have two potentially nonvanishing threepoint functions: $j=(-1,-1 / 2,-1 / 2)$ and $j=(-1,-1,-1)$. In the following, we investigate these two cases.

Let us start with general comments. The holography relation (5.12) suggests that the correlation functions of non-normalizable operators should be singular, with the poles corresponding to propagators of normalizable states [discrete $\mathrm{SL}(2, R) / \mathrm{U}(1)$ representations]. The residues are related to the correlation functions of normalizable operators. The latter correlation functions are finite.

The three-point correlation functions for the coset $\mathrm{SL}(2, \mathbb{R}) / \mathrm{U}(1)$ model were computed in [59], see also $[33,68]$. The pole structure can be investigated by the method used in [59]. (Note however that there is a typo in [[59] Eq. (4.20)]), see Appendix D).

\section{A. $j=(-1,-1 / 2,-1 / 2)$ correlation function}

In this subsection, we consider the three-point correlation function with one $j=-1$ operator and two $j=-1 / 2$ operators and also investigate its possible holographic relation to the correlation function with corresponding non-normalizable operators. We see that there is indeed such a relation in the $j=-1 \leftrightarrow \tilde{j}=0$ channel. Let us start with this one.

\section{A non-normalizable $\tilde{j}_{1}=0$ operator}

Consider three operators with $\operatorname{SL}(2, \mathbb{R})$ spins: $\tilde{j}_{1}=0, j_{2}=-1 / 2, j_{3}=-1 / 2$. The first one is nonnormalizable, and by (5.10), it should correspond to a normalizable operator with $j_{1}=-1$. Since the other two operators are not non-normalizable, they do not lead to poles in the three-point function, and the resulting correlation function contains only one pole. By using the technique of [59] (see Appendix A 2 b for details), we arrive at the expression

$$
\begin{aligned}
& \left\langle V_{\tilde{j}_{1} ; m_{1},-m_{1}} V_{j_{2}=-1 / 2 ; m_{2},-m_{2}} V_{j_{3}=-1 / 2 ; m_{3},-m_{3}}\right\rangle \\
& =\frac{R_{m_{1} m_{2} m_{3}}}{\tilde{j}_{1}}+\text { (regular terms), }
\end{aligned}
$$

where we used that $m_{1} \in \mathbb{Z} \backslash\{0\}$ and $m_{2}, m_{3} \in 1 / 2+\mathbb{Z}$. We see that there is a pole at $\tilde{j}_{1}=0$. The residue is calculated to be

$$
R_{m_{1} m_{2} m_{3}}=\frac{1}{2 \pi},
$$

cf. (6.6) and the comments below.

\section{A normalizable $j=-1$ operator}

Now, we consider a similar correlation function, only this time with all fields normalizable, $j_{1}=-1, j_{2}=j_{3}=$ $-1 / 2$. Namely, consider the three-point correlation function

$$
\left\langle V_{j_{1}=-1 ; m_{1},-m_{1}} V_{j_{2}=-1 / 2 ; m_{2},-m_{2}} V_{j_{3}=-1 / 2 ; m_{3},-m_{3}}\right\rangle .
$$

Using the reflection property (5.4), we obtain the exact expression

$$
\begin{aligned}
& \left\langle V_{j_{1}=-1 ; m_{1},-m_{1}} V_{j_{2} ; m_{2},-m_{2}} V_{j_{3} ; m_{3},-m_{3}}\right\rangle \\
& \quad=\frac{\left\langle V_{\tilde{j}_{1}=0 ; m_{1},-m_{1}} V_{j_{2} ; m_{2},-m_{2}} V_{j_{3} ; m_{3},-m_{3}}\right\rangle}{R\left(\tilde{j}_{1}=0, m_{1},-m_{1} ; k\right)} .
\end{aligned}
$$

As we saw above, both the numerator and denominator have poles at $\tilde{j}_{1}=-1-j_{1}=0$. Substituting (6.3) and (5.14), here we get

$$
\begin{aligned}
& \left\langle V_{j_{1}=-1 ; m_{1},-m_{1}} V_{j_{2}=-1 / 2 ; m_{2},-m_{2}} V_{j_{3}=-1 / 2 ; m_{3},-m_{3}}\right\rangle \\
& =\frac{\underset{\tilde{j}_{1}=0}{\operatorname{Res}}\left\langle V_{\tilde{j}_{1} ; m_{1},-m_{1}} V_{j_{2}=-1 / 2 ; m_{2},-m_{2}} V_{j_{3}=-1 / 2 ; m_{3},-m_{3}}\right\rangle}{\underset{\tilde{j}_{1}=0}{\operatorname{Res}} R\left(\tilde{j}_{1}, m_{1},-m_{1} ; k\right)} \\
& =\frac{1}{m_{1}^{2}} \text {. }
\end{aligned}
$$


This correlation function is finite. Note that the value $m_{1}=$ 0 is excluded by (3.22).

To conclude this subsection, let us note that the correlation function (6.4) describes a decay of one 4D physical state into two other physical states. Clearly, the conservation of 4D momentum and mass-shell conditions for all three states ensure that the heaviest state can decay into two other states only if its mass is larger than the sum of masses of other states. For example, the state with $j=-1$ can decay into two states from the $j=-1 / 2$ series if its mass is larger then the sum of the masses of the two $j=-1 / 2$ states,

$\operatorname{Mass}_{j_{1}=-1, m_{1}} \geq \operatorname{Mass}_{j_{2}=-1 / 2, m_{2}}+\operatorname{Mass}_{j_{3}=-1 / 2, m_{3}}$.

Using mass formulas (3.26) and (3.29) and the winding conservation (6.1), we arrive at

$$
\begin{aligned}
m_{1}^{2} & \geq m_{2}^{2}-\frac{1}{4}+m_{3}^{3}-\frac{1}{4}+2 \sqrt{\left(m_{2}^{2}-\frac{1}{4}\right)\left(m_{3}^{3}-\frac{1}{4}\right)} \\
& \Leftrightarrow m_{2} m_{3} \geq-\frac{1}{4} .
\end{aligned}
$$

In our case, this is equivalent to the condition that $m_{2}$ and $m_{3}$ are of the same sign. If this condition is not satisfied, we have the heaviest $j=-1 / 2$ state decaying to a lighter $j=$ $-1 / 2$ state and a $j=-1$ state. Figure 1 summarizes these results.

\section{3. $\phi e^{-Q / 2 \phi}$ and would-be $L S Z$ poles}

In Sec. VC3, we considered the non-normalizable operator with $j=-1 / 2$. We saw that the two-point function for such an operator has a double pole.

It turns out that the three-point function with insertions of the logarithmic primaries (5.20) has an unexpected structure. This three-point function is calculated in Appendix $2 \mathrm{~b}$. The result is

$$
\begin{aligned}
& \left\langle V_{\tilde{j}_{1}=0, m,-m} \phi V_{\tilde{j}_{2}=-1 / 2, m,-m} \phi V_{\tilde{j}_{3}=-1 / 2, m,-m}\right\rangle \\
& \quad=\frac{1}{2 \pi} \frac{1}{\tilde{j}_{1}\left(\tilde{j}_{2}+\frac{1}{2}\right)\left(\tilde{j}_{3}+\frac{1}{2}\right)} .
\end{aligned}
$$

This is not the expected LSZ behavior. From Eqs. (1.1) and (5.23), one could expect that the three-point correlation function (6.9) has a single pole corresponding to $\tilde{j}_{1}=0$ and two double poles corresponding to $\tilde{j}_{2}, \tilde{j}_{3}=-1 / 2$. The single pole at $\tilde{j}_{1}=0$ shows up in Eq. (6.9) as expected, but the other two are single rather than double poles. What is going on?

Moreover, single poles at $\tilde{j}_{2}, \tilde{j}_{3}=-1 / 2$ in Eq. (6.9) are not LSZ poles. Indeed, according to [39], when we consider the $x_{i}$-dependent correlation function and integrate over $x_{i}$ [cf. (A4)], the LSZ poles are seen as poles coming from

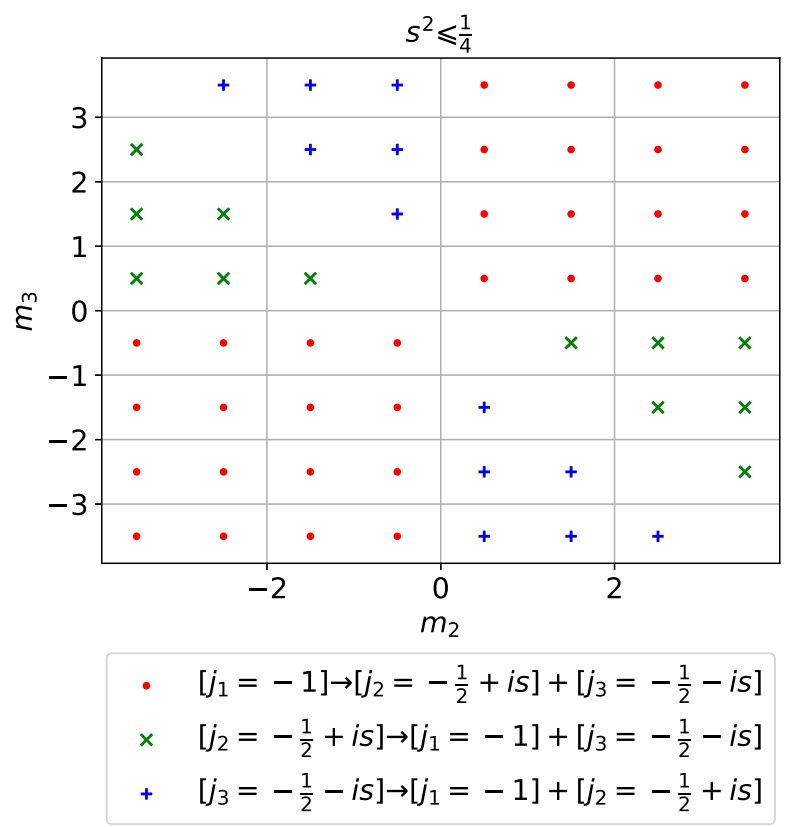

FIG. 1. Possible decays determined by the energy conservation. The quantum numbers $j, m$ are as they appear in the three-point correlation function (7.6). The $\left(m_{2}, m_{3}\right)$ plane extends infinitely. Note that the points with $m_{2}=-m_{3}$ are excluded because of winding conservation (6.1) and the fact that for $j_{1}=-1$ it is required that $m_{1} \neq 0$ (3.29).

$x_{i} \rightarrow 0, \infty$. However, this integrated correlation function also exhibits poles that can be traced back to coinciding $x_{i}$ under the integral [and also to $x \rightarrow 1$ in the expression (A4)]. The analysis shows that the single $j=-1 / 2$ poles in (6.9) are exactly of this type, see Appendix A 2 a for details.

In other words, these are "bulk poles" coming from the bulk of the cigar, i.e., from the region of large $\phi$ rather than LSZ poles, which come from states localized near the tip of the cigar.

We conclude that the holography picture does not work for the $j=-1 / 2$ channel.

\section{B. $j=(-1,-1,-1)$ correlation function}

Consider three operators with spins $j_{1}=j_{2}=j_{3}=-1$. Corresponding non-normalizable operators should have $\tilde{j}_{1}=\tilde{j}_{2}=\tilde{j}_{3}=0$.

In this case, one could expect that the correlation function of non-normalizable operators with $\tilde{j}=0$ should contain three poles, but it does not. This can be seen by analyzing the exact formula for the three-point function (A1). The coefficient $D$ from (A3) becomes

$$
\begin{aligned}
& D=\frac{k}{2 \pi^{3}}\left[\frac{1}{\pi} \frac{\Gamma\left(1+\frac{1}{k}\right)}{\Gamma\left(1-\frac{1}{k}\right)}\right]^{j_{1}+j_{2}+j_{3}+1} \frac{\Gamma\left(1-\frac{j_{1}+j_{2}+j_{3}+1}{k}\right)}{\Gamma\left(\frac{j_{1}+j_{2}+j_{3}+1}{k}\right)} \\
& \stackrel{j_{i} \rightarrow 0, k \rightarrow 1}{\longrightarrow} \frac{k}{2 \pi^{4}} .
\end{aligned}
$$


The factor (A4) cannot produce all the necessary poles, and the correlation function under consideration does not have the necessary pole structure. See also a discussion in Appendix B of [59].

Similarly, we can check that the three-point function of three normalizable operators with $j=-1$ is zero. Although this time the coefficient $D$ in Eq. (A3) happens to contain a single pole, the function $F\left(j_{1}, m_{1} ; j_{2}, m_{2} ; j_{3}, m_{3}\right)$ in Eq. (A4) has a double zero unless $m_{1}=m_{2}=0$ [59]; however, the latter values of $m$ are excluded, see (3.22). Therefore, the whole correlation function vanishes,

$$
\left\langle V_{j_{1}=-1 ; m_{1},-m_{1}} V_{j_{2}=-1 ; m_{2},-m_{2}} V_{j_{3}=-1 ; m_{3},-m_{3}}\right\rangle=0 .
$$

This completes the analysis of the three-point correlation functions of vertex operators corresponding to baryons of the $4 \mathrm{D} \mathcal{N}=2$ SQCD.

\section{CONTINUOUS REPRESENTATIONS}

So far, we have discussed only the vertex operators from the discrete representations (3.17), as they have direct interpretations as hadrons of the underlying $4 \mathrm{D} \mathcal{N}=2$
SQCD [17,18]. However, there is also the principal and exceptional continuous representations.

In this section, we concentrate on the vertex operators from the principal continuous representation with ${ }^{12}$ $j=-1 / 2+i s$, see (3.18). The scaling dimensions of these vertex operators are given by

$$
\Delta=m^{2}-\frac{1}{4}+s^{2} .
$$

The parameter $s \in \mathbb{R}$ here is continuous, which leads to a continuous mass spectrum for the states created by these vertex operators. This prevents interpretation of such states as baryons in 4D SQCD. It was argued in [17] that these string states are multiparticle states related to the presence of massless bifundamental quarks in 4D SQCD. To shed more light on their nature, below we study correlation functions with insertions of such operators.

\section{A. Two-point correlation function}

The two-point correlation function of such states is given by

$$
\begin{aligned}
\left\langle V_{j_{1}}=-1 / 2+i s ; m_{L}, m_{R}\right. & \left.V_{j_{2}=-1 / 2+i s ;-m_{L},-m_{R}}\right\rangle=R\left(-1 / 2+i s, m_{L}, m_{R} ; k\right) \\
& =\left[\frac{1}{\pi} \frac{\Gamma\left(1+\frac{1}{k}\right)}{\Gamma\left(1-\frac{1}{k}\right)}\right]^{2 i s} \frac{\Gamma\left(1-\frac{2 i s}{k}\right) \Gamma\left(1 / 2+i s+m_{L}\right) \Gamma\left(1 / 2+i s+m_{R}\right) \Gamma(-2 i s)}{\Gamma\left(1+\frac{2 i s}{k}\right) \Gamma\left(m_{L}+1 / 2-i s\right) \Gamma\left(1 / 2-i s+m_{R}\right) \Gamma(2 i s)} \\
& =\left[\frac{1}{\pi} \frac{\Gamma\left(1+\frac{1}{k}\right)}{\Gamma\left(1-\frac{1}{k}\right)}\right]^{2 i s} \cdot e^{i \delta\left(s ; m_{L}, m_{R} ; k\right)},
\end{aligned}
$$

where we have introduced a phase $\delta\left(s ; m_{L}, m_{R} ; k\right)$. This correlation function does not have poles in the variable $s$; instead, it should be interpreted simply as a scattering phase, see, e.g., Ref. [62]. The only possible problem with Eq. (7.2) is that now the limit $k \rightarrow 1$ is not well defined because of the first factor. However, this factor is nonphysical and can be absorbed into the definition of vertex operators (5.3).

Finally, let us comment on the factor $\delta\left(j_{1}-j_{2}\right)$ coming from (5.9). It does not enter the formula for the two-point function (7.2) for the following reason. The states with $j=$ $-1 / 2+i s$ form a continuum. When we calculate an amplitude with such states, we should integrate over all final states, that is,

$$
\begin{aligned}
& \int d s \delta\left(s-s^{\prime}\right)\left\langle V_{j_{1}=-1 / 2+i s ; m_{L}, m_{R}} V_{j_{2}=-1 / 2+i s^{\prime} ;-m_{L},-m_{R}}\right\rangle \\
& =\left\langle V_{j_{1}=-1 / 2+i s ; m_{L}, m_{R}} V_{j_{2}=-1 / 2+i s ;-m_{L},-m_{R}}\right\rangle .
\end{aligned}
$$

\footnotetext{
${ }^{12}$ On the interpretation of $\operatorname{Im}\{j\}$ as a momentum along the cigar, see, e.g., [17,69-71].
}

The two-point function of $j=-1 / 2$ operators considered in Sec. V C 2 can be considered as the limiting $s \rightarrow 0$ case of the correlation function considered here. Therefore, the delta function factor is absent in that expression, too.

\section{B. Three-point correlation function}

In Sec. VIA, we derived the three-point function with $j=(-1,-1 / 2,-1 / 2)$. That derivation can be easily adopted for the three-point function with continuous representation, which turns out to be nontrivial in the case ${ }^{13}$

$j_{1}=-1 \leftrightarrow \tilde{j}_{1}=0 ; \quad j_{2}=-\frac{1}{2}+i s, \quad j_{3}=-\frac{1}{2}-i s$.

The three-point function in this case is as follows (see Appendix A 2 a for a detailed computation):

$$
\begin{gathered}
\left\langle V_{\tilde{j}_{1} \rightarrow 0 ; m_{1},-m_{1}} V_{-1 / 2+i s ; m_{2},-m_{2}} V_{-1 / 2-i s ; m_{3},-m_{3}}\right\rangle \\
=\frac{1}{\tilde{j}_{1}} \times \frac{1}{2 \pi} e^{i\left(\delta\left(s ; m_{2},-m_{2} ; k\right)-\delta\left(s ; m_{3},-m_{3} ; k\right)\right)} .
\end{gathered}
$$

\footnotetext{
${ }^{13}$ The case with $j_{3}=-\frac{1}{2}+i s$ is also nontrivial but can be recovered from (7.4) using the reflection property (5.4).
} 
The phase factor $\delta$ is defined in (7.2). In the limit $s \rightarrow 0$, in the three-point function (7.5), the phase goes to zero, and we recover the answer (6.2) with the residue given by (6.3).

We see that the three-point correlation function with one non-normalizable operator with $\tilde{j}=0$ again has a single pole. This confirms the LSZ holography relation (5.12) for correlation functions of operators from principal continuous representation. The corresponding three-point function with normalizable operators is going to be finite; it can be calculated by the same method as in Sec. VIA2. The answer is

$$
\left\langle V_{-1 ; m_{1},-m_{1}} V_{-1 / 2+i s ; m_{2},-m_{2}} V_{-1 / 2-i s ; m_{3},-m_{3}}\right\rangle=\frac{1}{m_{1}^{2}} e^{i\left(\delta\left(s ; m_{2},-m_{2} ; k\right)-\delta\left(s ; m_{3},-m_{3} ; k\right)\right)}
$$

Much in the same way as for states from the discrete spectrum the decay of the $j=-1$ state into two states from the $j=-\frac{1}{2} \pm i s$ series can go only if the mass of the decaying particle is greater than the sum of the masses of the products,

$$
\operatorname{Mass}_{j_{1}=-1, m_{1}} \geq \operatorname{Mass}_{j_{2}=-1 / 2+i s, m_{2}}+\operatorname{Mass}_{j_{3}=-1 / 2-i s, m_{3}} .
$$

Using mass formulas (7.1) and (3.29) and the winding conservation (6.1), we arrive at

$$
\begin{aligned}
m_{1}^{2} & \geq m_{2}^{2}+s^{2}-\frac{1}{4}+m_{3}^{3}+s^{2}-\frac{1}{4}+2 \sqrt{\left(m_{2}^{2}+s^{2}-\frac{1}{4}\right)\left(m_{3}^{3}+s^{2}-\frac{1}{4}\right)} \\
& \Leftrightarrow m_{2} m_{3}-s^{2}+\frac{1}{4} \geq \sqrt{\left(m_{2}^{2}+s^{2}-\frac{1}{4}\right)\left(m_{3}^{3}+s^{2}-\frac{1}{4}\right)} \\
& \Leftrightarrow\left\{\begin{array}{l}
s^{2} \leq \frac{1}{4} \\
m_{2}, m_{3}
\end{array} \text { are of same sign } .\right.
\end{aligned}
$$

The condition that the $j_{1}=-1$ state decays into two continuous with $j_{2,3}=-1 / 2 \pm i$ s turns out to be

$$
\left\{\begin{array}{l}
s^{2} \leq \frac{1}{4} \\
m_{2}, m_{3} \text { are of same sign }
\end{array}\right.
$$

Figure 1 summarizes these results. Note that decay via a three particle process (when one particles decays to two) is possible if and only if when it involves either all discrete states or two continuous with $s^{2} \leq 1 / 4$. A decay via a three particle process cannot involve continuous states with $s^{2}>1 / 4$.

\section{Interpretation of continuous representations}

The correlation function (7.6) that we just computed corresponds to a process where a $j=-1$ state decays into two conjugate states,

$$
[j=-1] \rightarrow\left[j=-\frac{1}{2}+i s\right]+\left[j=-\frac{1}{2}-i s\right],
$$

cf. the discussion of operator mixing in [62].

How can we interpret the states corresponding to the principal continuous representation (3.18)? As we already mentioned, according to (7.1) their energies form a continuous spectrum. However, in $4 D$ SQCD, we do not expect to have a family of hadrons with continuous distribution of masses.

In [17], it was suggested that these states can be interpreted as decaying modes of normalizable physical 4D states. This was confirmed by showing that spectra of continues states start from thresholds given by masses (3.26) and (3.29). Here, we can confirm this interpretation for the case of principal continuous representation.

Moreover, we can further specify this: a $j=-1 / 2+i s$ state can be interpreted as a multipartical state of a $j=$ $-1 / 2$ baryon and massless bifundamental quarks. Then, the decay (7.10) corresponds to the decay of a $j=-1$ state into two $j=-1 / 2$ states (which we know can happen from Sec. VI A) with the radiation of massless bifundamental quarks.

\section{CONCLUSIONS}

In this paper, we studied correlation functions in the string theory for the critical non-Abelian vortex. Specifically, we considered these correlation functions using the equivalent description in terms of string theory on the $\mathrm{SL}(2, \mathbb{R}) / \mathrm{U}(1)$ WZNW coset and studied their analytic structure.

We also compared our solitonic string-gauge duality, which relates hadrons of $4 \mathrm{D} \mathcal{N}=2 \mathrm{SQCD}$ to closed string states of the string theory of the critical non-Abelian vortex 
with the AdS/CFT approach. Suggesting that the solitonic string-gauge duality can be thought of as a "no branes" limit of the AdS/CFT, we tested the holography, which is a distinctive feature of the AdS/CFT correspondence.

We showed that the AdS/CFT-type holography relation (1.1) found for LST [39] or Eq. (5.12) is fulfilled in our theory for most channels. This relation ensures that correlation functions of non-normalizable operators in the string theory on the cigar correspond to correlation functions of normalizable operators, which in turn are directly associated with hadronic states in $4 \mathrm{D} \mathcal{N}=2$ SQCD. The only exception are operators of the $j=$ $-1 / 2$ series, which are on the borderline between normalizable and non-normalizable operators and correspond to physical states in 4D SQCD. It turns out that in the $j=$ $-1 / 2$ channel holography relation does not work.

Technically, the reason for the holography relation (1.1) is that non-normalizable and normalizable operators with the same conformal dimension are related by the reflection of the tip of the cigar. It would be interesting to understand a deep conceptual reason behind this holography.

We can also mention several other open questions. The first is to find a more direct relation between critical string theory on the conifold and noncritical $c=1$ string theory with the Liouville field. The second is the construction of the effective theory of hadron interactions in the 4D $\mathcal{N}=2$
SQCD. In principle, it should be possible using results obtained in this paper for correlation functions in the string theory. They fix the coupling constants of the effective interactions of hadrons in SQCD. The symmetry restrictions, especially the $\mathcal{N}=2$ supersymmetry, heavily restrict the form of possible interaction terms. Moreover, the interpretation of continuous representations deserves more solid justification. We leave these problems for future work.

\section{ACKNOWLEDGMENTS}

The authors are grateful to M. Shifman for valuable discussions. The work of A. Y. was supported by the William I. Fine Theoretical Physics Institute, University of Minnesota, and Skolkovo Institute of Science and Technology.

\section{APPENDIX A: THREE-POINT FUNCTIONS}

Now let us review the results for the three-point functions in the supersymmetric Liouville theory.

\section{Full three-point function formula}

The full analytical expression for the three-point function of the operators $V_{j, m,-m}$ was derived in [59]. Let us write it down as

$$
\begin{aligned}
\left\langle V_{\tilde{j}_{1} ; m_{1},-m_{1}} V_{j_{2} ; m_{2},-m_{2}} V_{j_{3} ; m_{3},-m_{3}}\right\rangle= & D\left(\tilde{j}_{1}, j_{2}, j_{3} ; k\right) \\
& \times F\left(\tilde{j}_{1}, m_{1} ; j_{2}, m_{2} ; j_{3}, m_{3}\right) \int d^{2} x|x|^{2\left(m_{1}+m_{2}+m_{3}-1\right)} .
\end{aligned}
$$

The last factor here simply enforces the baryon charge conservation

$$
m_{1}+m_{2}+m_{3}=0
$$

The first factor ${ }^{14}$ equals

$$
\begin{aligned}
D\left(\tilde{j}_{1}, j_{2}, j_{3} ; k\right)= & \frac{k}{2 \pi^{3}}\left[\frac{1}{\pi} \frac{\Gamma\left(1+\frac{1}{k}\right)}{\Gamma\left(1-\frac{1}{k}\right)}\right]^{\tilde{j}_{1}+j_{2}+j_{3}+1} \\
& \times \frac{G\left(-\tilde{j}_{1}-j_{2}-j_{3}-2\right) G\left(j_{3}-\tilde{j}_{1}-j_{2}-1\right) G\left(j_{2}-\tilde{j}_{1}-j_{3}-1\right) G\left(\tilde{j}_{1}-j_{2}-j_{3}-1\right)}{G(-1) G\left(-2 \tilde{j}_{1}-1\right) G\left(-2 j_{2}-1\right) G\left(-2 j_{3}-1\right)} .
\end{aligned}
$$

Some properties of the special $G$ function used here are listed in Appendix B 6. The $F$ factor in Eq. (A1) is given by

$$
\begin{aligned}
F\left(\tilde{j}_{1}, m_{1} ; j_{2}, m_{2} ; j_{3}, m_{3}\right)= & \int d^{2} x_{1} d^{2} x_{2}\left|x_{1}\right|^{2\left(\tilde{j}_{1}+m_{1}\right)}\left|x_{2}\right|^{2\left(j_{2}+m_{2}\right)} \\
& \times\left|1-x_{1}\right|^{2\left(j_{2}-\tilde{j}_{1}-j_{3}-1\right)}\left|1-x_{2}\right|^{2\left(\tilde{j}_{1}-j_{2}-j_{3}-1\right)}\left|x_{1}-x_{2}\right|^{2\left(j_{3}-\tilde{j}_{1}-j_{2}-1\right)} .
\end{aligned}
$$

\footnotetext{
${ }^{14}$ The structure constants (A3) are equivalent to (B22). They can be derived from (B22) using (B23) and slightly changing the normalization of the operators.
} 


\section{The case with continuous and discrete representations}

Now let us calculate the three-point functions (7.5) and (6.2).

$$
\text { a. } j=-1 / 2 \pm i s
$$

Now let us turn to the computation of the three-point function in the case (7.4),

$j_{1}=-1 \leftrightarrow \tilde{j}_{1}=0 ; \quad j_{2}=-\frac{1}{2}+i s, \quad j_{3}=-\frac{1}{2}-i s$.

We analyze the exact expression for the three-point function (A1). Substituting (A5) into (A3) and using (B24) and (B25), we obtain

$$
\begin{aligned}
& D\left(\tilde{j}_{1}, j_{2}, j_{3} ; k\right) \\
& =\frac{k}{2 \pi^{3}} \times \frac{G(-1) G(-2 i s-1) G(2 i s-1) G\left(\tilde{j}_{1}\right)}{G(-1) G(-1) G(-2 i s) G(2 i s)}+\ldots \\
& =\frac{k}{2 \pi^{3}}\left(-\frac{k}{\tilde{j}_{1}}\right)\left(-\frac{(2 i s)^{2}}{k^{2}}\right)+\ldots \\
& =-\frac{2 s^{2}}{\pi^{3}} \frac{1}{\tilde{j}_{1}}+\ldots,
\end{aligned}
$$

where the ellipses stand for the terms nonsingular at Eq. (7.4). Next, let us evaluate the integral factor A4. From Eq. (7.4), we see that $\tilde{j}_{1}-j_{2}-j_{3}-1=0$. Making a change of variables,

$$
x_{1}=x, \quad x_{2}=x t,
$$

we can rewrite $F\left(\tilde{j}_{1}, m_{1} ; j_{2}, m_{2} ; j_{3}, m_{3}\right)$ from Eq. (A4) as

$$
\begin{aligned}
F\left(\tilde{j}_{1}, m_{1} ; j_{2}, m_{2} ; j_{3}, m_{3}\right)= & \int d^{2} x d^{2} t|x|^{2\left(\tilde{j}_{1}+m_{1}\right)+2+2\left(j_{2}+m_{2}\right)+2\left(j_{3}-\tilde{j}_{1}-j_{2}-1\right)}|1-x|^{2\left(j_{2}-\tilde{j}_{1}-j_{3}-1\right)} \\
& \times|t|^{2\left(j_{2}+m_{2}\right)}|1-t|^{2\left(j_{3}-\tilde{j}_{1}-j_{2}-1\right)} .
\end{aligned}
$$

Using Eq. (A2), we obtain

$$
\begin{aligned}
F\left(\tilde{j}_{1}, m_{1} ; j_{2}, m_{2} ; j_{3}, m_{3}\right)= & \int d^{2} x d^{2} t|x|^{2\left(j_{3}-m_{3}\right)}|1-x|^{2\left(j_{2}-\tilde{j}_{1}-j_{3}-1\right)} \\
& \times|t|^{2\left(j_{2}+m_{2}\right)}|1-t|^{2\left(j_{3}-\tilde{j}_{1}-j_{2}-1\right)} .
\end{aligned}
$$

Finally, substituting (7.4) here gives

$$
\begin{aligned}
F\left(\tilde{j}_{1}, m_{1} ; j_{2}, m_{2} ; j_{3}, m_{3}\right)= & \int d^{2} x|x|^{2\left(-1 / 2-m_{3}-i s\right)}|1-x|^{2(2 i s-1)} \\
& \times \int d^{2} t|t|^{2\left(-1 / 2+m_{2}+i s\right)}|1-t|^{2(-2 i s-1)} .
\end{aligned}
$$

These integrals can be calculated with the help of (D1),

$$
\begin{aligned}
\int d^{2} x|x|^{2\left(-1 / 2-m_{3}-i s\right)}|1-x|^{2(2 i s-1)} & =\pi \frac{\Gamma\left(1 / 2-m_{3}-i s\right) \Gamma(2 i s) \Gamma\left(-i s+m_{3}+1 / 2\right)}{\Gamma\left(1 / 2+m_{3}+i s\right) \Gamma(1-2 i s) \Gamma\left(i s-m_{3}+1 / 2\right)} \\
& =\frac{i \pi}{2 s} \cdot \frac{\Gamma\left(1 / 2-m_{3}-i s\right) \Gamma(2 i s) \Gamma\left(-i s+m_{3}+1 / 2\right)}{\Gamma\left(1 / 2+m_{3}+i s\right) \Gamma(-2 i s) \Gamma\left(i s-m_{3}+1 / 2\right)} .
\end{aligned}
$$

Note that the pole at $s=0$ in this expression comes from the region $x \rightarrow 1$. According to [39], this means that this pole is the "bulk pole;" that is, it does not signify propagation of a physical state.

Putting all expressions together (and omitting the factor $\int d^{2} x|x|^{2\left(m_{1}+m_{2}+m_{3}-1\right)}$, which gives a conservation law for $m$ 's) we arrive at Eq. (7.5)

$$
\left\langle V_{\tilde{j}_{1} \rightarrow 0 ; m_{1},-m_{1}} V_{-1 / 2+i s ; m_{2},-m_{2}} V_{-1 / 2-i s ; m_{3},-m_{3}}\right\rangle=\frac{1}{\tilde{j}_{1}} \times \frac{1}{2 \pi} e^{i\left(\delta\left(s ; m_{2},-m_{2} ; k\right)-\delta\left(s ; m_{3},-m_{3} ; k\right)\right)} .
$$


Finally, we note that one can check the pole structure of the resulting correlation function (A12) by performing the calculation differently. Namely, we can first study the singularity at $x_{1} \rightarrow 0$ in Eq. (A4) which would give the pole at $\tilde{j}_{1}+m_{1}=-1,-2, \ldots$. The results are consistent.

One could worry that the pole at $\tilde{j}=0$ might mix with a so-called bulk pole (see the discussion in Sec. 2.4 of Ref. [39]). This can happen in the case when all but one vertex operator in a correlator are normalizable, but one operator has a normalizable and a non-normalizable part, both of which contribute to a pole. However, in our case, the operator with $\tilde{j}=0$ has no bulk contribution, since

$$
\begin{aligned}
V_{\tilde{j}_{1} \approx 0 ; m_{1},-m_{1}} & \sim e^{Q \cdot 0 \cdot \phi}+R\left(\tilde{j}_{1} \approx 0, m_{1},-m_{1} ; k\right) \cdot e^{-Q \phi} \\
& \sim \operatorname{Id}+R\left(\tilde{j}_{1} \approx 0, m_{1},-m_{1} ; k\right) \cdot e^{-Q \phi},
\end{aligned}
$$

where Id is the so-called "fake identity" operator, see, e.g., [[72] Sec. 12.2.2]. The second term here represents a normalizable operator times a pole. Plugging (A13) into the three-point correlator, we see that the second term reproduces the pole (A12), while the first term with Id gives the two-point function of two $j=-1 / 2 \pm i$ s operators, which is finite and does not contain a pole.

$$
\text { b. } j=-1 / 2
$$

The three-point function in the case with discrete representations $\tilde{j}_{1}=0, j_{2}=j_{3}=-1 / 2 \quad$ (6.2) can be obtained by formally sending $s$ to zero in the previous calculation. Setting $s=0$ in Eq. (7.5), we recover Eqs. (6.2) and (6.3).

Moreover, careful examination of the limit $j_{1} \rightarrow$ $-1, j_{2} \rightarrow-1 / 2, j_{3} \rightarrow-1 / 2$ in Eq. (A1) shows that the three-point function (6.4) is indeed finite, cf. (6.6). Setting $s=0$ in Eq. (7.6), we recover Eq. (6.6).

Another interesting question is the three-point function with two logarithmic primary fields,

$$
\left\langle V_{\tilde{j}_{1}=0, m,-m} \phi V_{\tilde{j}_{2}=-1 / 2, m,-m} \phi V_{\tilde{j}_{3}=-1 / 2, m,-m}\right\rangle .
$$

This three-point function can be derived using the results of Appendix A 2 a. To do this, we recall that, first, in the nonsupersymmetric Liouville theory, we have

$$
\phi e^{Q \phi}=-\left.i \frac{\partial}{\partial s} e^{2\left(\frac{Q}{2}+i s\right) \phi}\right|_{s=0},
$$

and second that an analytic continuation of the three-point function in the nonsupersymmetric Liouville gives the structure constants $D\left(\tilde{j}_{1}, j_{2}, j_{3} ; k\right)$ (A3) in the supersymmetric Liouville. From this, we can conclude that we can obtain the three-point function (A14) as follows ${ }^{15}$ :

\footnotetext{
${ }^{15}$ There are also logarithmic terms coming from the worldsheet dependence like in (B31), but these are subleading.
}

$$
\begin{aligned}
& \left\langle V_{\tilde{j}_{1}=0, m,-m} \phi V_{\tilde{j}_{2}=-1 / 2, m,-m} \phi V_{\tilde{j}_{3}=-1 / 2, m,-m}\right\rangle \\
& =\left\{\left[\frac{\partial}{\partial s_{1}} \frac{\partial}{\partial s_{2}} D\left(0,-1 / 2+i s_{1},-1 / 2-i s_{2} ; k\right)\right]\right. \\
& \quad \times F\left(0, m_{1} ;-1 / 2+i s_{1}, m_{2} ;-1 / 2-i s_{2}, m_{3}\right) \\
& \left.\quad \times \int d^{2} x|x|^{2\left(m_{1}+m_{2}+m_{3}-1\right)}\right\}\left.\right|_{s_{1}=s_{2}=0} .
\end{aligned}
$$

Performing the calculation, we obtain

$$
\begin{aligned}
& \left\langle V_{\tilde{j}_{1}=0, m,-m} \phi V_{\tilde{j}_{2}=-1 / 2, m,-m} \phi V_{\tilde{j}_{3}=-1 / 2, m,-m}\right\rangle \\
& =\frac{1}{2 \pi} \frac{1}{\tilde{j}_{1}\left(j_{2}+\frac{1}{2}\right)\left(j_{3}+\frac{1}{2}\right)} .
\end{aligned}
$$

\section{APPENDIX B: COMPARISON TO THE NONSUPERSYMMETRIC LIOUVILLE THEORY}

In this paper, we mainly consider the $\operatorname{SL}(2, \mathbb{R}) / \mathrm{U}(1)$ coset WZNW model, which is a mirror of the $\mathcal{N}=2$ Liouville theory. However, it is interesting to compare with the results in nonsupersymmetric Liouville theory. From that, we can draw lessons for our case [for example, why do we see a specific combination of exponentials in Eq. (5.1)] and find out some factorization properties.

Discussing various Liouville models below we need to introduce the Liouville $b$ parameter. So in order not to confuse it with our VEV of the massless baryon in 4D SQCD, we denote the Liouville parameter of the nonsupersymmetric theory as $b_{L}$ and in the supersymmetric model as $b_{\mathcal{N}=2}$. Moreover, we denote the background charges in nonsupersymmetric Liouville, supersymmetric Liouville, and $\operatorname{SL}(2, \mathbb{R}) / \mathrm{U}(1)$ coset WZNW, respectively, as $Q_{L}, Q_{\mathcal{N}=2}$, and $Q_{\mathrm{SL}}$.

\section{Non-SUSY vs. SUSY}

In the nonsupersymmetric Liouville theory (considered, e.g., in $[31,32,73])$, the interaction term is

$$
\mathcal{L}_{\text {int }} \sim e^{2 b_{L} \phi_{L}}
$$

The requirement that this is a marginal deformation (of conformal weight 1 ) leads to

$$
b_{L}\left(Q_{L}-b_{L}\right)=1 \Rightarrow Q_{L}=b_{L}+\frac{1}{b_{L}},
$$

see also Ref. [36] [Eq. (2.13)].

On the other hand, in the $\mathcal{N}=2$ Liouville, the interaction term can be written as

$$
\mathcal{L}_{\text {int }} \sim \int d^{2} \theta e^{-\frac{1}{\sqrt{2}} b_{\mathcal{N}=2}\left(\phi_{\mathcal{N}=2}+i Y\right)} .
$$


(Here, we use the normalization where the asymptotic radius of the compact dimension is $R=\sqrt{2 / k}$, see Sec. III.) In components, we have terms like $b_{\mathcal{N}=2}^{2} \psi^{+} \psi^{-} e^{b_{\mathcal{N}=2}\left(\phi_{\mathcal{N}=2}+i Y\right)}$. The requirement that this is a marginal deformation (of conformal weight 1 ) leads to

$$
\begin{gathered}
\frac{1}{2}+\frac{b_{\mathcal{N}=2}}{2 \sqrt{2}}\left(Q_{\mathcal{N}=2}-\frac{b_{\mathcal{N}=2}}{2 \sqrt{2}}\right)+\frac{b_{\mathcal{N}=2}^{2}}{8} \\
=1 \Rightarrow Q_{\mathcal{N}=2}=\frac{\sqrt{2}}{b_{\mathcal{N}=2}}
\end{gathered}
$$

see also [36] [Eq. (11.7)]. That is why (B3) can be written as

$$
\mathcal{L}_{\text {int }} \sim \int d^{2} \theta e^{-\frac{\phi_{\mathcal{N}=2+i Y}}{Q_{\mathcal{N}=2}}}
$$

cf. [39] [Eq. (3.9)] and [17] [Eq. (4.8)].

Next, consider the $\mathrm{SL}(2, \mathbb{R}) / \mathrm{U}(1)$ theory, which is a mirror to the $\mathcal{N}=2$ Liouville [20,21,25,26]. In this model, the asymptotic radius is $R=\sqrt{2 k}$. The vertex operators at large positive $\phi$ are written as (see Sec. V for details)

$$
V \sim e^{Q_{\mathrm{SL}} j \phi+Q_{\mathrm{SL}} i m Y}
$$

Here,

$$
Q_{\mathrm{SL}}=Q_{\mathcal{N}=2}=\sqrt{\frac{2}{k}}
$$

\section{The dictionary}

Extending the dictionary from Teschner's papers [34] [Eq. (17)], [33] [Sec. IV. 4] (see also Ref. [36] [Eq. (6.92) and below]), we can write the correspondence ${ }^{16}$ between quantities in supersymmetric and nonsupersymmetric Liouville theories,

$$
\begin{aligned}
b_{L} & \leftrightarrow \frac{1}{\sqrt{k}}, \\
\alpha & \leftrightarrow-b_{L} j, \\
Q_{L} & \leftrightarrow \frac{1}{\sqrt{2}} Q_{\mathrm{SL}},
\end{aligned}
$$

where $\alpha$ defines the primary operator $\exp \left\{2 \alpha \phi_{L}\right\}$ in the Liouville theory. (Also note that the level $k$ in Teschner's papers is shifted by 2.) Comparing Eqs. (B4), (B7), and (B8), we conclude that $b_{\mathcal{N}=2}=\frac{1}{b_{L}}$, and

\footnotetext{
${ }^{16}$ In $[74,75]$, an alternative dictionary was presented.
}

$$
Q_{\mathrm{SL}}=\sqrt{2} b_{L} .
$$

This matches with [36] [Eq. (6.100)], [76] [Eq. (2.2)].

For example, according to Eqs. (B8) and (B9), the map acts as follows:

$$
\begin{aligned}
& \alpha=Q_{L} / 2 \leftrightarrow j=-\frac{1}{b} \frac{1}{\sqrt{2}} Q_{\mathrm{SL}} / 2=-1 / 2, \\
& Q_{L}-\alpha \leftrightarrow-\frac{1}{b}\left(\frac{1}{\sqrt{2}} Q_{\mathrm{SL}}+b_{L} j\right)=-1-j .
\end{aligned}
$$

Moreover, the Liouville field is mapped as

$$
\phi_{L}=-\frac{1}{\sqrt{2}} \phi_{\mathcal{N}=2}
$$

\section{Stress tensor}

The stress tensor is mapped correctly,

$$
\begin{aligned}
T_{L} & =-\left(\partial \phi_{L}\right)^{2}+Q_{L} \partial^{2} \phi_{L} \\
& \rightarrow-\left(\partial \phi_{L}\right)^{2}+\frac{1}{\sqrt{2}} Q_{\mathrm{SL}} \partial^{2} \phi_{L} \\
& =-\frac{1}{2}\left[\left(\partial \phi_{\mathcal{N}=2}\right)^{2}+\sqrt{\frac{2}{k}} \partial^{2} \phi_{\mathcal{N}=2}\right],
\end{aligned}
$$

which is the $\phi$ part of the full stress tensor (3.3). Primary operators (in the large- $\phi$ limit) and conformal dimensions are also mapped correctly,

$$
\begin{aligned}
e^{2 \alpha \phi_{L}} & \leftrightarrow e^{2\left(-\frac{j}{\sqrt{k}}\right)\left(-\frac{1}{\sqrt{2}} \phi_{\mathcal{N}=2}\right)}=e^{\sqrt{2 / k j} j \phi_{\mathcal{N}=2}}=e^{Q_{\mathrm{SL}} j \phi_{\mathcal{N}=2}}, \\
\Delta & =\alpha\left(Q_{L}-\alpha\right) \leftrightarrow-b_{L} j\left(\frac{1}{\sqrt{2}} Q_{\mathrm{SL}}+b j\right) \\
& =-b_{L}^{2} j(j+1)=-\frac{j(j+1)}{k} .
\end{aligned}
$$

Primary fields of the nonsupersymmetric Liouville theory $\sim e^{2 \alpha \phi}$ can be both normalizable and nonnormalizable.

\section{Reflection coefficient}

With this dictionary, the inverse ${ }^{17}$ reflection coefficient from $[31,32,73]$ correctly reproduces the $m$-independent part of the reflection coefficient (5.2). Indeed,

\footnotetext{
${ }^{17}$ Some authors define the reflection coefficient $R$ (schematically) as $V_{\alpha}=R(\alpha) V_{Q-\alpha}$, while others use the definition $V_{\alpha}=R(Q-\alpha) V_{Q-\alpha}$. The two reflection coefficients are reciprocal with respect to each other. Our conventions in the bulk of this paper (and also the conventions of [39]) correspond to the former definition, while the authors of $[32,73]$ use the latter.
} 


$$
\begin{aligned}
R(\alpha)_{L} & =\left[\pi \mu_{L} \gamma\left(b_{L}^{2}\right)\right]^{\left(2 \alpha-Q_{L}\right) / b_{L}} \frac{b_{L}^{2}}{\gamma\left(2 \alpha / b_{L}-1-1 / b_{L}^{2}\right) \gamma\left(2 b_{L} \alpha-b_{L}^{2}\right)} \\
& =\left[\pi \mu_{L} \gamma\left(b_{L}^{2}\right)\right]^{\left(2 \alpha-Q_{L}\right) / b_{L}} \frac{b_{L}^{2}}{\gamma\left(\frac{2 \alpha-Q_{L}}{b_{L}}\right) \gamma\left(b_{L}\left(2 \alpha-Q_{L}\right)+1\right)} \\
& \rightarrow\left[\pi \mu_{L} \gamma\left(b_{L}^{2}\right)\right]^{\left(2\left(-b_{L} j\right)-Q_{\mathrm{SL}}\right) / b_{L}} \frac{b_{L}^{2}}{\gamma\left(\frac{2\left(-b_{L} j\right)-Q_{\mathrm{SL}}}{b_{L}}\right) \gamma\left(b_{L}\left(2 \alpha-Q_{\mathrm{SL}}\right)+1\right)} \\
& =\left[\pi \mu_{L} \gamma\left(\frac{1}{k}\right)\right]^{-2 j-1} \frac{1}{k} \frac{1}{\gamma(-2 j-1) \gamma\left(1-\frac{2 j+1}{k}\right)} .
\end{aligned}
$$

Then,

$$
\begin{aligned}
\frac{1}{R(\alpha)_{L}} & \rightarrow k\left[\pi \mu_{L} \frac{\Gamma\left(\frac{1}{k}\right)}{\Gamma\left(1-\frac{1}{k}\right)}\right]^{2 j+1} \frac{\Gamma\left(1-\frac{2 j+1}{k}\right) \Gamma(-2 j-1)}{\Gamma\left(\frac{2 j+1}{k}\right) \Gamma(2 j+2)} \\
& =\left[k \pi \mu_{L} \frac{\Gamma\left(1+\frac{1}{k}\right)}{\Gamma\left(1-\frac{1}{k}\right)}\right]^{2 j+1} \frac{\Gamma\left(1-\frac{2 j+1}{k}\right) \Gamma(-2 j-1)}{\Gamma\left(1+\frac{2 j+1}{k}\right) \Gamma(2 j+1)} .
\end{aligned}
$$

On the other hand, the reflection coefficient in the supersymmetric Liouville theory is given by (5.2). One can see that that the analytically continued reflection coefficient in the nonsupersymmetric Liouville (B15) matches the $m$-independent part of the supersymmetric reflection amplitude (5.2) up to the factor $\left(k \pi^{2} \mu_{L}\right)^{2 j+1}$. (Recall that $m$ is the momentum along the compact direction, which is absent in the pure Liouville theory.) However, a factor of the form $(. .)^{2 j+1}$ is insignificant, since it can be absorbed into a redefinition of the operators (5.3).

\section{DOZZ formula}

The formula for the three-point correlation function in nonsupersymmetric pure Liouville theory (the so-called DOZZ formula) was derived in $[31,32]$. Later, it was extensively studied by many authors; one can mention $[73,77,78]$ and a review [36]. The structure constants $C\left(\alpha_{1}, \alpha_{2}, \alpha_{3}\right)$ [the analog of $D\left(j_{1}, j_{2}, j_{3} ; k\right)$ from the supersymmetric case (A3)] are

$$
\begin{aligned}
C\left(\alpha_{1}, \alpha_{2}, \alpha_{3}\right)= & {\left[\pi \mu \gamma\left(b_{L}^{2}\right) b_{L}^{2-2 b_{L}^{2}}\right]\left(Q_{L}-\sum \alpha_{i}\right) / b_{L} } \\
& \times \frac{\Upsilon_{0} \Upsilon\left(2 \alpha_{1}\right) \Upsilon\left(2 \alpha_{2}\right) \Upsilon\left(2 \alpha_{3}\right)}{\Upsilon\left(\alpha_{1}+\alpha_{2}+\alpha_{3}-Q_{L}\right) \Upsilon\left(\alpha_{1}+\alpha_{2}-\alpha_{3}\right) \Upsilon\left(\alpha_{2}+\alpha_{3}-\alpha_{1}\right) \Upsilon\left(\alpha_{1}+\alpha_{3}-\alpha_{2}\right)} .
\end{aligned}
$$

Here, $Q_{L}=b_{L}+1 / b_{L}$.

Let us review the special function $\Upsilon$, see also [32] [Eqs. (3.10-13)], [73] [Appendix A]. It can be defined as

$\log \Upsilon(x)=\int_{0}^{\infty} \frac{d t}{t}\left[\left(\frac{Q_{L}}{2}-x\right)^{2} e^{-t}-\frac{\sinh ^{2}\left(\frac{Q_{L}}{2}-x\right) \frac{t}{2}}{\sinh \frac{b_{L} t}{2} \sinh \frac{t}{2 b_{L}}}\right]$,

while

$$
\Upsilon_{0}=\left.\frac{d \Upsilon(x)}{d x}\right|_{x=0}
$$

Some useful properties of the $\Upsilon$ function are

$$
\begin{gathered}
\Upsilon(x)=\Upsilon\left(Q_{L}-x\right), \\
\Upsilon\left(Q_{L} / 2\right)=1, \\
\Upsilon\left(x+b_{L}\right)=\gamma\left(b_{L} x\right) b_{L}^{1-2 b_{L} x} \Upsilon(x), \\
\Upsilon\left(x+1 / b_{L}\right)=\gamma\left(x / b_{L}\right) b^{2 x / b_{L}-1} \Upsilon(x), \\
\Upsilon\left(x-b_{L}\right)=\gamma\left(b_{L} x-b_{L}^{2}\right)^{-1} b_{L}^{2 b_{L} x-1-2 b_{L}^{2}} \Upsilon(x),
\end{gathered}
$$$$
\Upsilon\left(x-1 / b_{L}\right)=\gamma\left(x / b_{L}-1 / b_{L}^{2}\right)^{-1} b_{L}^{1+\frac{2}{b_{L}^{2}}-\frac{2 x}{b_{L}}} \Upsilon(x) \text {. }
$$

Here, we use the standard notation

$$
\gamma(x)=\frac{\Gamma(x)}{\Gamma(1-x)} .
$$


The function $\Upsilon(x)$ has simple zeros at $x=-m b_{L}-n / b_{L}$ and $x=\left(m^{\prime}+1\right) b_{L}+\left(n^{\prime}+1\right) / b_{L}$. Here, $n, n^{\prime}, m, m^{\prime}=$ $0,1,2, \ldots$

\section{Different form of the structure constants in the supersymmetric Liouville theory}

The three-point function in a corresponding WZNW model (which is a mirror of the $\mathcal{N}=2$ Liouville) was calculated in [33,34], see also [59]. It is given by the product of the structure constants $D\left(j_{1}, j_{2}, j_{3} ; k\right)$ (which do not depend on the momentum along the compact dimension $m$ ) and an $m$-dependent part. It turns out that under the dictionary (B8), the structure constants for this supersymmetric Liouville theory (A3) maps to the three-point function of the nonsupersymmetric Liouville (B16).

\section{a. Structure constants}

In Ref. [33], the author considers the $\operatorname{SL}(2, \mathbb{C}) / \mathrm{SU}(2)$ WZNW model. ${ }^{18}$ The result for the three-point structure constants is [33] [Eq. (64)]

$$
C\left(\alpha_{1}, \alpha_{2}, \alpha_{3}\right)=\frac{C_{0}\left(b_{L}\right)\left(\nu\left(b_{L}\right)\right)^{-b_{L}^{-1}\left(\alpha_{1}+\alpha_{2}+\alpha_{3}\right)} \Upsilon\left(2 \alpha_{1}\right) \Upsilon\left(2 \alpha_{2}\right) \Upsilon\left(2 \alpha_{3}\right)}{\Upsilon\left(\alpha_{1}+\alpha_{2}+\alpha_{3}-b_{L}\right) \Upsilon\left(\alpha_{1}+\alpha_{2}-\alpha_{3}\right) \Upsilon\left(\alpha_{1}+\alpha_{3}-\alpha_{2}\right) \Upsilon\left(\alpha_{2}+\alpha_{3}-\alpha_{1}\right)}
$$

Here, $C_{0}\left(b_{L}\right)\left(\nu\left(b_{L}\right)\right)^{-b_{L}^{-1}\left(\alpha_{1}+\alpha_{2}+\alpha_{3}\right)}$ is a coefficient that can be found in Ref. [33].

Comparing this formula to the nonsupersymmetric case (B16), we see that the first $\Upsilon$ function in the denominator of (B22) depends on $b_{L}$ while in (B16) in the same place enters $Q_{L}$. This fits with our dictionary (B8) and (B9). One can also compare to [34] [Eq. (66)], [34] [Eq. (23)] (in the latter paper, the author uses a different normalization of operators, see [[34] last paragraph of Appendix A]).

The three-point function formula can be also written in terms of the special $G$ function. Such a form of the threepoint correlation function is quite popular in the literature, so let us review it here, see also [62] [Eqs. (2.15-18)], [59] [Eq. (A3)], [39] [Appendix A]. The function $G$ can be expressed via the Barnes double gamma function, see, e.g., [62] [Eq. (2.15)]. The relation between the $\Upsilon$ and $G$ functions is given by

$$
G(j)=b_{L}^{-b_{L}^{2} j\left(j+1+b_{L}^{-2}\right)} \frac{1}{\Upsilon\left(b_{L}(j+1)\right)}
$$

cf. [34] [between Eqs. (23) and (24)]. ${ }^{19}$ Let us list some useful properties of the $G$ function,

$$
\begin{aligned}
G(j) & =G(-j-1-k), \\
G(j-1) & =\gamma\left(1+\frac{j}{k}\right) G(j), \\
G(j-k) & =k^{-(2 j+1)} \gamma(j+1) G(j) .
\end{aligned}
$$

From (B24), we can derive another useful property,

\footnotetext{
${ }^{18}$ The manifold $\mathrm{SL}(2, \mathbb{C}) / \mathrm{SU}(2)$ can be thought of as a Euclidean version of $\operatorname{SL}(2, \mathbb{R})$.

${ }^{19}$ Note that Teschner uses in [34] a different notation for $j$, namely, $j_{\text {Teschner }}=-j_{\text {our }}-1$.
}

$$
\frac{G(j-1)}{G(j)} \frac{G(-j-1)}{G(-j)}=-\frac{j^{2}}{k^{2}} .
$$

The function $G(j)$ has poles at $j=n+m k$ and $j=-(n+1)-(m+1) k, n, m=0,1,2, \ldots$.

Using the relation (B23) and that $\alpha=-b_{L} j$ [see the dictionary (B8)], one can easily see that the structure constants formulas (A3) and (B22) are equivalent.

\section{Factorization}

From what we have seen so far, we can make an observation. The three-point correlation function formula (A1) for the $\mathrm{SL}(2, \mathbb{R}) / \mathrm{U}(1)$ (supersymmetric Liouville) theory can be naturally split into a product of "structure constants" that do not depend on the compact momentum $m$ and an $m$-dependent part. Moreover, it turns out that the structure constants in the supersymmetric theory (A3) and (B22) precisely correspond to the analytically continued structure constants in the nonsupersymmetric Liouville (B16), when we use the dictionary (B8) and (B9).

So we see a curious property. The two- and three-point correlation functions in the supersymmetric Liouville factorize into the (analytically continued) "nonsupersymmetric Liouville" part and an extra $m$-dependent part. For the two-point function, it was noted in [33]. In [74], an alternative prescription was presented.

\section{Two-point function from the DOZZ formula}

In this section, we are going to show that the two-point function with non-normalizable $\tilde{j}=-1 / 2$ operators (5.20),

$$
\left\langle\phi V_{\tilde{j}=-1 / 2, m,-m} \phi V_{\tilde{j}=-1 / 2, m,-m}\right\rangle,
$$

has a double pole. To this end, we are going to use the factorization property, see the previous subsection. According to this property, the $m$-dependent part of the correlation function (B26) is given simply by the 
$m$-dependent part of the reflection amplitude (5.2), while for the $m$-independent part we can take the analytically continued result from the Liouville theory.

So let us consider for a moment the correlation function,

$$
\left\langle\phi e^{2 \alpha \phi_{L}} \phi e^{2 \alpha \phi_{L}}\right\rangle, \quad \alpha=Q_{L} / 2,
$$

in the nonsupersymmetric Liouville theory. One way to compute this is to use the property ${ }^{20} \partial_{j} e^{Q j \phi} \sim \phi e^{Q j \phi}$.
Let us start from the identity [31,32,73] (see also Sec. 9.2.1 of Ref. [72]),

$$
2 \pi \delta\left(\alpha_{1}-\alpha_{2}\right)\left\langle e^{2 \alpha_{1} \phi_{L}} e^{2 \alpha_{2} \phi_{L}}\right\rangle=\lim _{\varepsilon \rightarrow 0}\left\langle e^{2 \alpha_{1} \phi_{L}} e^{2 \varepsilon \phi_{L}} e^{2 \alpha_{2} \phi_{L}}\right\rangle .
$$

The rhs here can be computed explicitly using the formulas from Appendix B 5.

Taking

$$
\alpha_{1}=\frac{Q_{L}}{2}+i p_{1}, \quad \alpha_{2}=\frac{Q_{L}}{2}+i p_{2}, \quad p_{1}, p_{2} \rightarrow 0, \quad \varepsilon \rightarrow 0,
$$

we get (here we do write explicitly dependence on the world-sheet coordinates),

$$
\begin{aligned}
\left\langle e^{2 \alpha_{1} \phi_{L}} e^{2 \varepsilon \phi_{L}} e^{\left.2 \alpha_{2} \phi_{L}\right\rangle \approx}\right. & \frac{2 \varepsilon}{\varepsilon^{2}+\left(p_{1}-p_{2}\right)^{2}} \frac{4 p_{1} p_{2}}{\varepsilon^{2}+\left(p_{1}+p_{2}\right)^{2}} \\
& \times \frac{1}{\left|z_{1}-z_{2}\right|^{2\left(\Delta_{1}+\Delta_{2}-\Delta_{\varepsilon}\right)}\left|z_{1}-z_{\varepsilon}\right|^{2\left(\Delta_{1}-\Delta_{2}+\Delta_{\varepsilon}\right)}\left|z_{2}-z_{\varepsilon}\right|^{2\left(-\Delta_{1}+\Delta_{2}+\Delta_{\varepsilon}\right)}} \approx 2 \pi \delta\left(p_{1}-p_{2}\right) \frac{\left(p_{1}+p_{2}\right)^{2}}{\varepsilon^{2}+\left(p_{1}+p_{2}\right)^{2}} \\
& \times \frac{1}{\left|z_{1}-z_{2}\right|^{2\left(\Delta_{1}+\Delta_{2}-\Delta_{\varepsilon}\right)}\left|z_{1}-z_{\varepsilon}\right|^{2\left(\Delta_{1}-\Delta_{2}+\Delta_{\varepsilon}\right)}\left|z_{2}-z_{\varepsilon}\right|^{2\left(-\Delta_{1}+\Delta_{2}+\Delta_{\varepsilon}\right)}},
\end{aligned}
$$

where $\Delta_{i}=\alpha_{i}\left(Q_{L}-\alpha_{i}\right)=Q_{L}^{2} / 4+p_{i}^{2}, \Delta_{\varepsilon}=\varepsilon\left(Q_{L}-\varepsilon\right)$, and $z_{1}, z_{2}, z_{\varepsilon}$ refer to the world-sheet coordinates of the corresponding operators.

To obtain the two-point function (B27), we need to take derivatives of (B30) with respect ${ }^{21}$ to $p_{1}, p_{2}$. However, this time, we should take into account the dependence on the world-sheet coordinates, since conformal dimensions depend on $p_{1}, p_{2}$. Taking the derivatives and dropping the $\delta^{\prime}, \delta^{\prime \prime}$ terms, we obtain

$$
\begin{aligned}
& \left\langle\phi e^{2 \alpha_{1} \phi_{L}} e^{2 \varepsilon \phi_{L}} \phi e^{2 \alpha_{2} \phi_{L}}\right\rangle \\
& \approx 2 \pi \delta\left(p_{1}-p_{2}\right) \frac{2 \varepsilon^{2}\left(\varepsilon^{2}-12 p_{1}^{2}\right)}{\left(\varepsilon^{2}+4 p_{1}^{2}\right)^{3}} \frac{1}{\left|z_{1}-z_{2}\right|^{4 \Delta}} \\
& \quad \times 2 \pi \delta\left(p_{1}-p_{2}\right) \frac{4 p_{1}^{2}}{\varepsilon^{2}+4 p_{1}^{2}} \frac{\ln \left|z_{1}-z_{2}\right|}{\left|z_{1}-z_{2}\right|^{4 \Delta}} .
\end{aligned}
$$

The delta function lets us to set $p_{1}=p_{2}$.

When we send $\varepsilon \rightarrow 0$, the first term here generically vanishes. However, if $p_{1}$ and $p_{2}$ both tend to zero, the first term becomes singular. From this, we see that the two-point function (B28) in fact has a double pole,

\footnotetext{
${ }^{20}$ Note that $\left.\partial_{j} V_{j, m_{L}, m_{R}}\right|_{j=-1 / 2}$ vanishes, cf. (5.1) and Sec. V C 2.

${ }^{21}$ These are easiest to compute in terms of the variables $p_{+}=p_{1}+p_{2}, p_{-}=p_{1}-p_{1}$.
}

$$
\left\langle\phi e^{-2 \alpha_{1} \phi} \phi e^{-2 \alpha_{2} \phi}\right\rangle \sim \delta\left(\alpha_{1}-\alpha_{2}\right) \frac{1}{\left(\alpha_{1}-Q_{L} / 2\right)^{2}} .
$$

From Eqs. (B32) and (5.19), we conclude that the full correlation function (B26) is given by

$$
\left\langle\phi V_{\tilde{j}_{1} \rightarrow-1 / 2, m,-m} \phi V_{\tilde{j}_{2} \rightarrow-1 / 2, m,-m}\right\rangle \sim \frac{1}{\left(\tilde{j}_{1}+\frac{1}{2}\right)^{2}} \delta\left(\tilde{j}_{1}-\tilde{j}_{2}\right) .
$$

\section{Pure Liouville degenerate operators}

One may wonder why in the primary operators (5.1) we see only the specific combination

$$
e^{Q_{\mathcal{N}=2} j \phi_{\mathcal{N}=2}}+R\left(j, m_{L}, m_{R} ; k\right) e^{-Q_{\mathcal{N}=2}(j+1) \phi_{\mathcal{N}=2}},
$$

while the orthogonal combination (with the minus sign in front of $R$ ) does not appear. We can see the hint of this in nonsupersymmetric Liouville theory.

The reflection property in the nonsupersymmetric Liouville theory $[31,32]$ can be written as

$$
e^{2\left(Q_{L}-\alpha\right) \phi_{L}}=R(\alpha)_{L} e^{2 \alpha \phi_{L}} .
$$

(See footnote 17 on page 60.) The two-point function is structurally similar to (5.9), 


$$
\left\langle e^{2 \alpha \phi_{L}} e^{2 \alpha \phi_{L}}\right\rangle \sim \frac{1}{R(\alpha)_{L}} .
$$

The formula (B34) should be interpreted as a valid relation on the level of correlation functions. For example, using Eqs. (B34) and (B35), one can see that

$$
\left\langle e^{2(Q-\alpha) \phi} e^{2 \alpha \phi}\right\rangle=R(\alpha)\left\langle e^{2 \alpha \phi} e^{2 \alpha \phi}\right\rangle=1 .
$$

Moreover,

$$
\begin{aligned}
& \left\langle\left(e^{2\left(Q_{L}-\alpha\right) \phi_{L}}-R(\alpha)_{L} e^{2 \alpha \phi_{L}}\right)\right. \\
& \left.\cdot\left(e^{2\left(Q_{L}-\alpha\right) \phi_{L}}-R(\alpha)_{L} e^{2 \alpha \phi_{L}}\right)\right\rangle=0 .
\end{aligned}
$$

Therefore, the combination $e^{2\left(Q_{L}-\alpha\right) \phi_{L}}-R(\alpha)_{L} e^{2 \alpha \phi_{L}}$ is in fact a null state, and in this sense, we can understand (B34). Of course, the reflection coefficient $R(\alpha)_{L}$ is different from (5.2), but they are closely related, see Appendix B. 4.

\section{APPENDIX C: TWO-POINT CORRELATION FUNCTION CHECK}

In this appendix, we use a quasiclassical approximation to check the consistency of the two-point function formula (5.9). Looking at Eqs. (5.1), (5.8), and (5.9) together, one may wonder whether these formulas are consistent. Namely, we would like to compare the pole structure of the rhs and lhs of (5.9).

Consider a correlation function of two non-normalizable operators. Suppose we use the expansion (5.1) in the two-point function (5.9) and go at one of the poles of $R(j, m,-m ; k)$. Then, it seems that in the rhs of (5.9) we get a single pole, which is fine for a two-point function. However, according to (5.1) and (5.8), on the lhs, both operators develop a pole, ${ }^{22}$ and it seems that we should get a double pole. What is going on?

To resolve this issue, it is easier to make the calculation of the correlation function in the Euclidean $\mathrm{AdS}_{3}$ theory with primary operators $\Phi_{j ; m_{L}, m_{R}}$ (it is equal to the correlation function of the coset operators, see, e.g., [59] [Eq. (3.4)]). Large $\phi$ expansion of these operators is similar to $(5.9)$,

$$
\begin{gathered}
\Phi_{j ; m_{L}, m_{R}} \approx e^{Q j \phi} \gamma^{j+m_{L}} \bar{\gamma}^{j-m_{R}}+R\left(j, m_{L}, m_{R} ; k\right) \\
\cdot e^{-Q(j+1) \phi} \gamma^{m_{L}-j-1} \bar{\gamma}^{-m_{R}-j-1} .
\end{gathered}
$$

Here, $(\phi, \gamma, \bar{\gamma})$ are the Poincaré coordinates on Euclidean $\mathrm{AdS}_{3}$ [39] [Eq. (2.14)]. The two-point function of these operators is the same as for the $V$ operators, see, e.g., [59] [Eqs. (3.4) and (3.6)],

\footnotetext{
${ }^{22}$ It is important that on the one hand (3.17) is symmetric in $m \leftrightarrow-m$, and on the other hand, $R(j, m,-m ; k)$ has the same poles as $R(j,-m, m ; k)$.
}

$\left\langle\Phi_{j_{1} ; m_{L}, m_{R}} \Phi_{j_{2} ;-m_{L},-m_{R}}\right\rangle=R\left(j_{1}, m_{L}, m_{R} ; k\right) \delta\left(j_{1}-j_{2}\right)$.

Let us concentrate on the case of interest $m_{L}=-m_{R} \equiv m$. Recall that the reflection coefficient $R(j, m,-m ; k)$ (5.2) has poles at special values of $j, m$ corresponding to discrete representations (3.17).

When calculating the correlation function of two operators (C1), we have to integrate over the (zero modes of) $\gamma$ 's. Moreover, the $\gamma$ 's in the first operator $\Phi_{j ; m,-m}$ and in the second operator $\Phi_{j ;-m, m}$ in (C2) are basically the same, since $\gamma(z) \gamma\left(z^{\prime}\right) \sim \gamma^{2}(z)\left(1+O\left(z-z^{\prime}\right)\right.$ ) (here, $z, z^{\prime}$ are the world-sheet coordinates).

Then, from $(\mathrm{C} 1)$ we have

$$
\begin{aligned}
\left\langle\Phi_{j_{1} ; m,-m} \Phi_{j_{2} ;-m, m}\right\rangle \sim & \int d^{2} \gamma\left\{\left\langle e^{Q j_{1}} e^{Q j_{2}}\right\rangle|\gamma|^{2\left(j_{1}+j_{2}\right)}\right. \\
& +\left\langle e^{Q j_{1}} e^{-Q\left(j_{2}+1\right)}\right\rangle|\gamma|^{-2} R\left(j_{2},-m, m ; k\right) \\
& +\left\langle e^{-Q\left(j_{1}+1\right)} e^{Q j_{2}}\right\rangle|\gamma|^{-2} R\left(j_{1}, m,-m ; k\right) \\
& +\left\langle e^{-Q\left(j_{1}+1\right)} e^{-Q\left(j_{2}+1\right)}\right\rangle|\gamma|^{-2\left(j_{1}+j_{2}+2\right)} \\
& \left.\times R\left(j_{1}, m,-m ; k\right) R\left(j_{2},-m, m ; k\right)\right\}
\end{aligned}
$$

Now, we need to integrate over $\gamma$. Using the integration formula (D1), we obtain

$$
\int_{\mathbb{C}}|\gamma|^{-2 a} d^{2} \gamma=\pi \frac{\Gamma(1-a) \Gamma(1) \Gamma(a-1)}{\Gamma(a) \Gamma(0) \Gamma(2-a)} .
$$

But this is zero, unless $a=1$ ! The divergence at $a=1$ can be interpreted as the volume of the target space, cf., e.g., [21] [below Eq. (3.5)], [62] [Eq. (5.12)].

From this, we see that there are two cases: either $j_{1}+j_{2}=-1$ or $j_{1}+j_{2} \neq-1$. Let us start with the latter. Integrating over $\gamma$ in (C3), we obtain

$$
\begin{aligned}
\left\langle\Phi_{j_{1} ; m,-m} \Phi_{j_{2} ;-m, m}\right\rangle \sim & \left\langle e^{Q j_{1}} e^{-Q\left(j_{2}+1\right)}\right\rangle R\left(j_{2},-m, m ; k\right) \\
& +\left\langle e^{-Q\left(j_{1}+1\right)} e^{Q j_{2}}\right\rangle R\left(j_{1}, m,-m ; k\right) .
\end{aligned}
$$

(The divergent $\gamma$ integral cancels in the $\operatorname{SL}(2, \mathbb{R}) / \mathrm{U}(1)$ coset theory, see [75] [Sec. III. 2].) Thus, the last term of (C3) (containing a would-be double pole) drops out. Now, it is time to say something about correlation functions of pure exponentials. They do not contain any $m$ or $\gamma$ dependence. Therefore, these correlation functions can be computed using the results from nonsupersymmetric Liouville theory, see Appendix B. From this logic, we obtain [cf. (B36)]

$$
\left\langle e^{-Q\left(j_{1}+1\right)} e^{Q j_{2}}\right\rangle=\delta\left(j_{1}-j_{2}\right) \text { in the vicinity } j_{1} \approx j_{2} .
$$

Using this in (C5), we arrive at 


$$
\left\langle\Phi_{j_{1} ; m,-m} \Phi_{j_{2} ; m,-m}\right\rangle \sim \delta\left(j_{1}-j_{2}\right) R\left(j_{1}, m,-m ; k\right) .
$$

This has the same pole structure as the formula (C2).

To conclude, we comment on the case $j_{1}=j_{2}=-1 / 2$. In this case, all the terms in (C3) do contribute. However, they all give comparable contributions, since the reflection coefficient does not actually develop a pole. This is consistent with the results of Sec. V C 2.

\section{APPENDIX D: USEFUL FORMULAS}

Integration formula useful for the $m$-mode expansion of $\operatorname{SL}(2, \mathbb{R})$ primary operators (see, e.g., Refs. $[59,62]$ ),

$$
\int_{\mathbb{C}} d^{2} x|x|^{2 a} x^{n}|1-x|^{2 b}(1-x)^{m}=\pi \frac{\Gamma(a+n+1) \Gamma(b+m+1) \Gamma(-a-b-1)}{\Gamma(-a) \Gamma(-b) \Gamma(a+b+m+n+2)}, \quad n, m \in \mathbb{Z} .
$$

This formula can be derived using the method outlined in the book by Green, Schwarz, Witten [79] (Secs. 7.2.2-7.2.3 there). When the integral in the lhs does not converge, we use the rhs as the analytic continuation.

Another useful formula that helps with derivations of Sec. VI: integrating by parts, one can show that

$$
\operatorname{Res}_{\epsilon \rightarrow 0} \int_{0}^{\infty} d x x^{-1+\epsilon} \cdot f(x)=f(0),
$$

at least if $f$ is differentiable and $f(x \rightarrow \infty)=0$. (However, this result seems to hold even if $f$ has singularities and is divergent at infinity.)

The residue (5.14) can be calculated as follows. Consider $\tilde{j}=\epsilon \ll 0$, and take half-integer $m>0$. We have

$$
\begin{aligned}
R(\tilde{j}=\epsilon, m,-m ; k) & =\left[\frac{1}{\pi} \frac{\Gamma\left(1+\frac{1}{k}\right)}{\Gamma\left(1-\frac{1}{k}\right)}\right]^{2 \epsilon+1} \frac{\Gamma\left(1-\frac{2 \epsilon+1}{k}\right) \Gamma(m+\epsilon+1) \Gamma(-m+\epsilon+1) \Gamma(-2 \epsilon-1)}{\Gamma\left(1+\frac{2 \epsilon+1}{k}\right) \Gamma(m-\epsilon) \Gamma(-m-\epsilon) \Gamma(2 \epsilon+1)} \\
& \approx \frac{1}{\pi}\left[\frac{1}{\pi} \frac{\Gamma\left(1+\frac{1}{k}\right)}{\Gamma\left(1-\frac{1}{k}\right)}\right]^{2 \epsilon} \frac{\Gamma(m+1) \Gamma(-m+\epsilon+1) \Gamma(-2 \epsilon-1)}{\Gamma(m) \Gamma(-m-\epsilon) \Gamma(1)} \\
& \approx \frac{1}{\pi} m \cdot \frac{\Gamma(-m+\epsilon+1) \Gamma(-2 \epsilon-1)}{\Gamma(-m-\epsilon)} .
\end{aligned}
$$

Next, we use the formula

$$
\Gamma(-n+\epsilon) \approx \frac{(-1)^{n}}{\epsilon n !} .
$$

Then,

$$
\begin{aligned}
R(\tilde{j}=\epsilon, m,-m ; k) & \approx \frac{1}{\pi} m \cdot \frac{\Gamma(-m+\epsilon+1) \Gamma(-2 \epsilon-1)}{\Gamma(-m-\epsilon)} \\
& \approx \frac{1}{\pi} m \cdot \frac{(-1)^{m-1}}{\epsilon(m-1) !} \cdot \frac{(-1)^{1}}{(-2 \epsilon)(1) !} \cdot \frac{(-\epsilon) m !}{(-1)^{m}} \\
& =\frac{m^{2}}{2 \pi} \cdot \frac{1}{\epsilon} .
\end{aligned}
$$

Finally, let us also note a typo in Ref. [59]. Using the formulas from this appendix and from Appendix A one can check that factorial signs in the numerator in the last line of Eq. (4.20) in [59] should not be there. So, the formula (4.20) in [59] should read

$$
\begin{aligned}
S(2 ; 3)= & \sum_{n=}^{\max \left\{N, n_{3}-1\right\}}\left(\begin{array}{l}
N \\
n
\end{array}\right) \frac{(-1)^{n_{3}-1-n}}{\left(n_{3}-1-n\right) !\left(n_{2}-1+n-N\right) !} \\
& \times \prod_{i=0}^{n_{3}-n-2}\left(2 j_{3}+n_{3}+N-n-i\right) \prod_{i=0}^{n_{2}+n-N-2}\left(2 j_{2}+n_{2}+n-i\right) .
\end{aligned}
$$


[1] A. Hanany and D. Tong, Vortices, instantons and branes, J. High Energy Phys. 07 (2003) 037.

[2] R. Auzzi, S. Bolognesi, J. Evslin, K. Konishi, and A. Yung, Non-Abelian superconductors: Vortices and confinement in $\mathcal{N}=2$ SQCD, Nucl. Phys. B673, 187 (2003).

[3] M. Shifman and A. Yung, Non-Abelian string junctions as confined monopoles, Phys. Rev. D 70, 045004 (2004).

[4] A. Hanany and D. Tong, Vortex strings and four-dimensional gauge dynamics, J. High Energy Phys. 04 (2004) 066.

[5] A. Abrikosov, On the magnetic properties of superconductors of the second group, Russian original-Zh. Eksp. Teor. Fiz. 32, 1442 (1957) Sov. Phys. JETP 5, 1174 (1957); H. Nielsen and P. Olesen, Vortex-line models for dual strings, Nucl. Phys. B61, 45 (1973); Reprinted in Solitons and Particles, edited by C. Rebbi and G. Soliani (World Scientific, Singapore, 1984), p. 365].

[6] D. Tong, TASI lectures on solitons, arXiv:hep-th/0509216.

[7] M. Eto, Y. Isozumi, M. Nitta, K. Ohashi, and N. Sakai, Solitons in the Higgs phase: The moduli matrix approach, J. Phys. A 39, R315 (2006).

[8] M. Shifman and A. Yung, Supersymmetric solitons and how they help us understand non-Abelian gauge theories, Rev. Mod. Phys. 79, 1139 (2007); . For an expanded version see Supersymmetric Solitons (Cambridge University Press, Cambridge, England, 2009).

[9] D. Tong, Quantum vortex strings: A review, Ann. Phys. (Amsterdam) 324, 30 (2009).

[10] M. Shifman and A. Yung, Critical string from nonAbelian vortex in four dimensions, Phys. Lett. B 750, 416 (2015).

[11] P. Fayet and J. Iliopoulos, Spontaneously broken supergauge symmetries and Goldstone spinors, Phys. Lett. B 51, 461 (1974).

[12] P. Candelas and X. C. de la Ossa, Comments on conifolds, Nucl. Phys. B342, 246 (1990).

[13] A. Neitzke and C. Vafa, Topological strings and their physical applications, arXiv:hep-th/0410178.

[14] P. Koroteev, M. Shifman, and A. Yung, Non-Abelian vortex in four dimensions as a critical string on a conifold, Phys. Rev. D 94, 065002 (2016).

[15] P. Koroteev, M. Shifman, and A. Yung, Studying critical string emerging from non-Abelian vortex in four dimensions, Phys. Lett. B 759, 154 (2016).

[16] E. Ievlev, M. Shifman, and A. Yung, String baryon in fourdimensional $\mathcal{N}=2$ supersymmetric QCD from the 2D-4D correspondence, Phys. Rev. D 102, 054026 (2020).

[17] M. Shifman and A. Yung, Critical non-Abelian vortex in four dimensions and little string theory, Phys. Rev. D 96, 046009 (2017).

[18] M. Shifman and A. Yung, Hadrons of $\mathcal{N}=2$ supersymmetric QCD in four dimensions from little string theory, Phys. Rev. D 98, 085013 (2018).

[19] D. Kutasov, Introduction to little string theory, in Superstrings and Related Matters 2001, Proceedings of the ICTP Spring School of Physics, edited by C. Bachas, K. S. Narain, and S. Randjbar-Daemi (2002), pp. 165-209, ISBN: 9295003-10-1, http://users.ictp.it/ pub_off/lectures/vol7.html.

[20] D. Ghoshal and C. Vafa, $c=1$ string as the topological theory of the conifold, Nucl. Phys. B453, 121 (1995).
[21] A. Giveon and D. Kutasov, Little string theory in a double scaling limit, J. High Energy Phys. 10 (1999) 034.

[22] A. Giveon, D. Kutasov, and O. Pelc, Holography for noncritical superstrings, J. High Energy Phys. 10 (1999) 035 .

[23] K. Hori and A. Kapustin, Duality of the fermionic 2-D black hole and $N=2$ Liouville theory as mirror symmetry, J. High Energy Phys. 08 (2001) 045.

[24] E. Witten, String theory and black holes, Phys. Rev. D 44, 314 (1991).

[25] S. Mukhi and C. Vafa, Two-dimensional black hole as a topological coset model of $c=1$ string theory, Nucl. Phys. B407, 667 (1993).

[26] H. Ooguri and C. Vafa, Two-dimensional black hole and singularities of CY manifolds, Nucl. Phys. B463, 55 (1996).

[27] L. J. Dixon, M. E. Peskin, and J. D. Lykken, $N=2$ superconformal symmetry and $\mathrm{SO}(2,1)$ current algebra, Nucl. Phys. B325, 329 (1989).

[28] P. M. S. Petropoulos, Comments on SU(1,1) string theory, Phys. Lett. B 236, 151 (1990).

[29] S. Hwang, Cosets as gauge slices in SU(1,1) strings, Phys. Lett. B 276, 451 (1992).

[30] J. M. Evans, M. R. Gaberdiel, and M. J. Perry, The no ghost theorem for $\operatorname{AdS}(3)$ and the stringy exclusion principle, Nucl. Phys. B535, 152 (1998).

[31] H. Dorn and H. J. Otto, Two and three point functions in Liouville theory, Nucl. Phys. B429, 375 (1994).

[32] A. B. Zamolodchikov and A. B. Zamolodchikov, Structure constants and conformal bootstrap in Liouville field theory, Nucl. Phys. B477, 577 (1996).

[33] J. Teschner, On structure constants and fusion rules in the SL $(2, \mathbb{C}) / \mathrm{SU}(2)$ WZNW model, Nucl. Phys. B546, 390 (1999).

[34] J. Teschner, Operator product expansion and factorization in the H+ (3) WZNW model, Nucl. Phys. B571, 555 (2000).

[35] R. Dijkgraaf, H. L. Verlinde, and E. P. Verlinde, String propagation in a black hole geometry, Nucl. Phys. B371, 269 (1992).

[36] Y. Nakayama, Liouville field theory: A decade after the revolution, Int. J. Mod. Phys. A 19, 2771 (2004).

[37] O. Aharony, S. Gubser, J. Maldacena, H. Ooguri, and Y. Oz, Large $N$ field theories, string theory and gravity, Phys. Rep. 323, 183 (2000).

[38] O. Aharony, M. Berkooz, D. Kutasov, and N. Seiberg, Linear dilatons, NS five-branes and holography, J. High Energy Phys. 10 (1998) 004.

[39] O. Aharony, A. Giveon, and D. Kutasov, LSZ in LST, Nucl. Phys. B691, 3 (2004).

[40] D. Tong, Monopoles in the Higgs phase, Phys. Rev. D 69, 065003 (2004).

[41] M. Shifman and A. Yung, Lessons from supersymmetry: Instead-of-Confinement mechanism, Int. J. Mod. Phys. A 29, 1430064 (2014).

[42] N. Seiberg and E. Witten, Monopoles, duality and chiral symmetry breaking in $N=2$ supersymmetric QCD, Nucl. Phys. B431, 484 (1994).

[43] P. Argyres, M. Plesser, and N. Seiberg, The moduli space of $\mathcal{N}=2$ SUSY QCD and duality in $\mathcal{N}=1$ SUSY QCD, Nucl. Phys. B471, 159 (1996). 
[44] For a review, see e.g. A. Achucarro and T. Vachaspati, Semilocal and electroweak strings, Phys. Rep. 327, 347 (2000).

[45] M. Shifman and A. Yung, Non-Abelian semilocal strings in $\mathcal{N}=2$ supersymmetric QCD, Phys. Rev. D 73, 125012 (2006).

[46] Minoru Eto, Jarah Evslin, Kenichi Konishi, Giacomo Marmorini, Muneto Nitta, Keisuke Ohashi, Walter Vinci, and Naoto Yokoi, On the moduli space of semilocal strings and lumps, Phys. Rev. D 76, 105002 (2007).

[47] M. Shifman, W. Vinci, and A. Yung, Effective world-sheet theory for non-Abelian semilocal strings in $\mathcal{N}=2$ supersymmetric QCD, Phys. Rev. D 83, 125017 (2011).

[48] D. Gepner, Space-time supersymmetry in compactified string theory and superconformal models, Nucl. Phys. B296, 757 (1988).

[49] T. Banks, L. J. Dixon, D. Friedan, and E. J. Martinec, Phenomenology and conformal field theory or can string theory predict the weak mixing angle?, Nucl. Phys. B299, 613 (1988).

[50] P. Argyres, M. R. Plesser, and A. Shapere, The Coulomb Phase of $\mathcal{N}=2$ Supersymmetric QCD, Phys. Rev. Lett. 75, 1699 (1995).

[51] K. Chandrasekharan, Elliptic Functions (Springer-Verlag, Berlin, 1985).

[52] N. Dorey, The BPS spectra of two-dimensional supersymmetric gauge theories with twisted mass terms, J. High Energy Phys. 11 (1998) 005.

[53] N. Dorey, T. J. Hollowood, and D. Tong, The BPS spectra of gauge theories in two and four dimensions, J. High Energy Phys. 05 (1999) 006.

[54] E. Gerchkovitz and A. Karasik, New vortex-string worldsheet theories from super-symmetric localization, J. High Energy Phys. 03 (2019) 090.

[55] J. M. Evans, M. R. Gaberdiel, and M. J. Perry, The no-ghost theorem and strings on $\mathrm{AdS}_{3}$, arXiv:hep-th/9812252; published in Proceedings of ICTP Spring School of Physics, Nonperturbative Aspects of Strings, Branes and Supersymmetry, edited by M. J. Duff et al. (World Scientific, River Edge, N.J., 1999), pp. 435-444.

[56] D. Kutasov and N. Seiberg, Non-critical superstrings, Phys. Lett. B 251, 67 (1990).

[57] A. Yung, Flux compactification for the critical non-Abelian vortex and quark masses, Phys. Rev. D 104, 025007 (2021).

[58] I. R. Klebanov and E. Witten, Superconformal field theory on threebranes at a Calabi-Yau singularity, Nucl. Phys. B536, 199 (1998).

[59] A. Giveon and D. Kutasov, Comments on double scaled little string theory, J. High Energy Phys. 01 (2000) 023.
[60] H. Erbin, J. Maldacena, and D. Skliros, Two-point string amplitudes, J. High Energy Phys. 07 (2019) 139.

[61] J. M. Maldacena and H. Ooguri, Strings in $\operatorname{AdS}(3)$ and $\mathrm{SL}(2, \mathbb{R})$ WZW model 1.: The spectrum, J. Math. Phys. (N.Y.) 42, 2929 (2001).

[62] J. M. Maldacena and H. Ooguri, Strings in $\operatorname{AdS}(3)$ and the $\mathrm{SL}(2, \mathbb{R})$ WZW model. Part 3. Correlation functions, Phys. Rev. D 65, 106006 (2002).

[63] N. Seiberg, Notes on quantum Liouville theory and quantum gravity, Prog. Theor. Phys. Suppl. 102, 319 (1990).

[64] V. Gurarie, Logarithmic operators in conformal field theory, Nucl. Phys. B410, 535 (1993).

[65] J. Nagi, Logarithmic primary fields in conformal and superconformal field theory, Nucl. Phys. B722, 249 (2005).

[66] R. Nivesvivat and S. Ribault, Logarithmic CFT at generic central charge: From Liouville theory to the $Q$-state Potts model, SciPost Phys. 10, 021 (2021).

[67] D. Kutasov and N. Seiberg, More comments on string theory on AdS(3), J. High Energy Phys. 04 (1999) 008.

[68] J. Teschner, Liouville theory revisited, Classical Quantum Gravity 18, R153 (2001).

[69] P. H. Ginsparg and G. W. Moore, Lectures on 2-D gravity and 2-D string theory, arXiv:hep-th/9304011.

[70] P. Di Francesco, P. Mathieu, and D. Senechal, Conformal Field Theory, 10.1007/978-1-4612-2256-9.

[71] J. M. Maldacena, H. Ooguri, and J. Son, Strings in AdS(3) and the $\mathrm{SL}(2, \mathbb{R}) \mathrm{WZW}$ model. Part 2. Euclidean black hole, J. Math. Phys. (N.Y.) 42, 2961 (2001).

[72] H. Erbin, Notes on 2d quantum gravity and Liouville theory, https://www.lpthe.jussieu.fr/ erbin/files/liouville_theory.pdf (2015).

[73] D. Harlow, J. Maltz, and E. Witten, Analytic continuation of Liouville theory, J. High Energy Phys. 12 (2011) 071.

[74] S. Ribault and J. Teschner, H + (3) - WZNW correlation functions from Liouville theory, J. High Energy Phys. 06 (2005) 014.

[75] C. M. Chang, Y. H. Lin, S. H. Shao, Y. Wang, and X. Yin, Little string amplitudes (and the unreasonable effectiveness of 6D SYM), J. High Energy Phys. 12 (2014) 176.

[76] S. Iguri and C. A. Nunez, Coulomb integrals for the SL $(2, \mathbb{R})$ WZW model, Phys. Rev. D 77, 066015 (2008).

[77] J. Teschner, On the Liouville three point function, Phys. Lett. B 363, 65 (1995).

[78] A. Kupiainen, R. Rhodes, and V. Vargas, The DOZZ formula from the path integral, J. High Energy Phys. 05 (2018) 094.

[79] M. B. Green, J. H. Schwarz, and E. Witten, Superstring Theory: 1 (Cambridge University Press, Cambridge, England, 1987). 